\title{
Dislocation motion and instability
}

\author{
Yichao Zhu* Stephen Jonathan Chapman* Amit Acharya ${ }^{\dagger}$
}

March 25, 2013

\begin{abstract}
The Peach-Koehler expression for the stress generated by a single (non-planar) curvilinear dislocation is evaluated to calculate the dislocation self stress. This is combined with a law of motion to give the self-induced motion of a general dislocation curve. A stability analysis of a rectilinear, uniformly translating dislocation is then performed. The dislocation is found to be susceptible to a helical instability, with the maximum growth rate occurring when the dislocation is almost, but not exactly, pure screw. The non-linear evolution of the instability is determined numerically, and implications for slip band formation and non-Schmid behaviour in yielding discussed.
\end{abstract}

Key Words: dislocation, Peach-Koehler, self-induced motion, mobility law, linear stability, asymptotic expansion, instability, slip band, non-associated flow.

\section{Introduction}

A fundamental, unsolved, and old question of plasticity theory is, as stated by Mott [25], "why the deformation of a crystalline substance is not uniformly distributed through it ..., but is localized in slip bands." The first observations revealing the details of slip band microstructures are those of Heidenreich and Shockley [17] and Brown [7] in pure Aluminium (which has high stacking fault energy and therefore no dislocation dissociation into partials). The basic observation is the occurrence of parallel slip planes 200-800 $\AA$ apart on which slip takes place. Brown [7] defines the traces of these planes on observation surfaces as elementary slip lines, the regions they separate as lamellae, and separated clusters of elementary slip lines as slip zones. Across each slip plane, the amount of slip (displacement discontinuity) is $\sim 2000 \AA$. In the early parts of deformation, these elementary slip lines cluster together in well-separated zones, which merge with the progress of deformation. To our knowledge, there does not exist a theoretical explanation of the phenomenon described above. In this paper we suggest one, with quantitative estimates of the thickness of the lamellae. In our

* Mathematical Institute, 24-29 St Giles, Oxford OX1 3LB

${ }^{\dagger}$ Civil \& Environmental Engineering, Carnegie Mellon University, Pittsburgh, PA 15213-3890 
picture, the phenomenon is linked to a generic, but crucially 3-d, instability of a single dislocation line which can cross-slip onto the primary slip plane when close to screw orientation. Interestingly, the criterion for instability involves more than the resolved shear stress on the primary plane in which slip progresses, analogous to the macroscopic phenomenon of non-Schmid behavior in yielding. In a sense to be made precise, the dynamics also selects the primary planes on which slip occurs to be the ones on which the resolved shear stress is maximised: this information is not put into the model a priori. Curiously, our prediction of slip band microstructure is truly dynamical in nature and independent of considerations of non-convex incremental energy minimisation as proposed by Ortiz and Repetto [26] and the large body of work engendered by it. The physical mechanism behind the Ortiz-Repetto model is strong latent hardening; ours is complementary to this picture, being based in cross-slip. We note here the earlier work on macroscopic strain localisation in single crystals via cross slip by Asaro and Rice [4], albeit for a different stage of deformation. Asaro and Rice [4] suggest, based on earlier work of Rice [28], that "there is strong theoretical basis for a wider exploration of cross-slip and similar processes as a basis for localization, via the destabilizing effect of non-normality." Based on our analysis of a particular dislocation instability, we contribute to this exploration by motivating a possible microscopic basis for such non-associated flow.

Chapman and Richardson $[14,13,15,29]$ present and discuss a vortex-density model of superconductivity with a conservation law for the vortex density which has strong similarities with the evolution equation for Nye tensor density in the Field Dislocation Mechanics (FDM) model of Acharya [1]. It is shown in these works of Chapman and Richardson that superconducting vortices are susceptible to a helical instability if a component of the driving force lies along the vortex line. Such a component produces no motion while the vortex is straight, but will cause any deviation to grow in time. Given the similarities between the vortex density model and FDM as far as the evolution of the vortex/dislocation density fields are concerned, it is natural to probe the existence of a similar instability in FDM. Despite appearances, it has long been clear that the equations of FDM, under appropriate assumptions, can reproduce the equations of motion of discrete dislocations; in the context of the vortex density model, Richardson [29] explicitly shows how to transform the evolution of a vortex within the density model as the evolution of a single curve. We utilise exactly the same idea in this paper, and our analysis, while motivated from the similarities between the vortex density model and FDM, can just as well be interpreted as an analysis of the 3-d Discrete Dislocation Method [23, 2]. Our initial thoughts were that dislocations may not be susceptible to the same helical instability except when climb is allowed (high temperature), since, unlike superconducting vortices, they are more or less required to move in a slip plane. However, we will see that the helical instability does manifest itself in the dislocation case, with the evolution proceeding via cross slip onto many slip planes.

The remainder of the paper is organised as follows. In Section 2 we review the Peach-Koehler expression for the force produced by a general curvilinear dislocation. We asymptotically expand this expression as the dislocation line is approached to find the local self force. We then introduce a law of motion which allows for but severely impedes climb. This device allows us to describe cross slip and the preferred active slip planes arise naturally as those 
with maximum resolved shear stress.

For analytical tractability, we do not include crystallographic constraints and require only that a point along the dislocation curve which is of edge character has a velocity vector in the plane spanned by its Burgers vector and the tangent direction to the curve. When it is close to screw orientation the velocity vector is allowed to be in any direction, i.e. during cross slip there is no preferred direction built into the model for the orientation of the cross slip plane and dislocation dynamics decides the plane to pick as well as the spacing between such planes. As mentioned earlier, the planes that are dynamically selected are ones on which the resolved shear stress is maximised, and it is perhaps natural to expect that this result will be preserved when the mobility law further constrains the available slip planes for cross-slip to be the set of crystallographically favoured ones. Of course, a bigger question is whether the instability is completely suppressed under the imposition of such a mobility law, and the inability to answer this question with definiteness is a current shortcoming of our work. Additionally, our model is effectively a line tension model; during non-linear evolution expanding loops on different slip planes are not spaced very far apart and it will be important to account for the full stress interaction between such portions of the dislocation line on different planes. It may be conjectured that this feature will add to the richness of the problem, perhaps allowing us to predict the spacing between slip zones (cf. Brown [7]) and on to the meso-macro phenomenon of coarse slip bands (Asaro [3], Bassani [5]).

In Section 3 we perform a linear stability analysis to determine the most unstable configurations and the maximal growth rate. In Section 4 we follow the instability into the non-linear regime numerically. Finally, in Section 5, we present physical interpretations of our mathematical results vis-à-vis dislocation slip band microstructure formation.

\section{Self-induced dislocation motion}

We limit ourselves to isotropic materials. The stress field of an arbitrary curved dislocation was first derived by Peach and Koehler in 1950 [27] by differentiating the components of displacement as given by Burgers in 1939 [8, 9]. Later Kröner [22], derived the same result by means of stress functions. The self-induced stress at a point $\mathbf{x}$ due to an an arbitrary curved dislocation is given by

$$
\boldsymbol{\sigma}=\boldsymbol{\sigma}^{1}+\left(\boldsymbol{\sigma}^{1}\right)^{T}+\boldsymbol{\sigma}^{2}
$$

where

$$
\begin{aligned}
\boldsymbol{\sigma}^{\mathbf{1}} & =\frac{\mu}{4 \pi} \int_{C}(\mathbf{b} \wedge \nabla) \frac{1}{Z} \otimes \mathrm{d} \mathbf{q}, \\
\boldsymbol{\sigma}^{2} & =\frac{\mu}{4 \pi(1-\nu)} \int_{C}(\mathrm{~d} \mathbf{q} \cdot(\mathbf{b} \wedge \nabla))\left(\nabla \otimes \nabla-\mathbf{I} \nabla^{2}\right) Z
\end{aligned}
$$

where $\mathbf{b}$ is the Burgers vector, $\mu$ is the shear modulus, $\nu$ is Poisson's ratio, $\mathbf{I}$ is the identity tensor, $\mathbf{q}$ is a the point on dislocation curve $C, \mathbf{z}=\mathbf{q}-\mathbf{x},|\mathbf{z}|=Z$, the products $\wedge$ and $\cdot$ are 
the cross (vector) and dot (scalar) products, $\otimes$ is the dyadic (tensor) product, and $\nabla$ is the gradient with respect to $\mathbf{q}$.

Since a dislocation has no mass, it cannot experience a Newtonian force. However, dislocations will move in the presence of an appropriate component of stress. If the total stress acting on the dislocation is $\boldsymbol{\sigma}$, then the work done by it can be thought of arising from a force per unit length along the dislocation line. With this interpretation Peach and Koehler [27] find the force per unit length to be given by

$$
\mathbf{f}=(\boldsymbol{\sigma} \cdot \mathbf{b}) \wedge \mathbf{l},
$$

where $\mathbf{l}$ is the unit tangential vector to the dislocation line.

The motion of a dislocation in response to this force is governed by the mobility law. For high speed dislocation motion this law will include inertial terms as well as dissipative or drag terms $[18]^{1}$. However, we consider here dislocations moving much more slowly than the elastic wave speed, for which the inertial term is negligible. In this case the law takes the general form

$$
\mathbf{v}=\mathbf{G}(\mathbf{f}) \text {, }
$$

for some mobility function $\mathbf{G}$; typically $\mathbf{G}$ will be zero for forces $\mathbf{f}$ below the Peierls barrier, and may grow linearly or nonlinearly above it. Here we suppose that the Peierls barrier is small enough to be neglected, and that the velocity law may be linearised for small applied forces. This assumption simplifies our discussion, though since our analysis is a perturbation analysis, the general conclusions should hold also for nonlinear force laws.

For an edge dislocation, slip is much easier than climb, so that the motion is effectively confined to the glide plane spanned by the tangent and Burgers vector, with a velocity approximately proportional to the force per unit length. However, for a screw dislocation the tangent vector and Burgers vector are parallel, so that it may have several glide planes depending on the crystal structure of the material. In the isotropic continuum model, the number of glide planes for a screw dislocation can be regarded as infinite; in effect any plane containing the tangent vector is a potential glide plane.

A common approximation takes the velocity of a dislocation to be proportional to the projection of the force per unit length onto its glide plane. Since a screw dislocation can have any glide plane, for a pure screw no projection is necessary. For an edge dislocation the projection tensor (operator) is $\mathbf{I}-\boldsymbol{\beta} \otimes \boldsymbol{\beta}$ where

$$
\beta=\frac{1 \wedge b}{|1 \wedge b|},
$$

is the unit normal to the glide plane (see Figure 1). Thus, if we write

$$
\mathbf{v}=\mathbf{M} \cdot \mathbf{f}
$$

\footnotetext{
${ }^{1}$ In fact, at very high speeds atomistic simulations show that dislocation motion becomes very rough and may result in spontaneous self-pinning and the production of large quantities of debris [24]
} 
where $\mathbf{M}$ is the mobility tensor, then

$$
\mathbf{M}= \begin{cases}m_{g}(\mathbf{I}-\boldsymbol{\beta} \otimes \boldsymbol{\beta}) & \text { edge, } \\ m_{g} \mathbf{I} & \text { screw, }\end{cases}
$$

where $m_{g}$ is the (constant) glide mobility, and is generally found to be of the order of $30 \mathrm{~m} \mathrm{MPa}^{-1} \mathrm{~s}^{-1}$ [19]. The actual value of $m_{g}$ is important only in so far as it sets the timescale for the dislocation motion; it does not affect the qualitative stability properties of dislocations.

An alternative law in which climb is allowed but difficult was given by [31], who wrote

$$
\mathbf{M}= \begin{cases}m_{g}(\mathbf{I}-\boldsymbol{\beta} \otimes \boldsymbol{\beta})+m_{c} \boldsymbol{\beta} \otimes \boldsymbol{\beta} & \text { edge } \\ m_{g} \mathbf{I} & \text { screw }\end{cases}
$$

where $m_{c}$ is the mobility constant for climb, with $m_{c} \ll m_{g}$. This definition resolves the force for an edge dislocation to its glide plane, but keeps the freedom of the motion of a screw dislocation. However, there are still difficulties with such a law, due to its singular nature: a pure screw has an isotropic mobility, yet if we add an infinitesimal edge component we change the mobility drastically. Thus for a curved dislocation on which there is a single point of pure screw with the remainder mixed, the mobility will have a discontinuity at that point. The behaviour of the law then crucially depends on the numerical discretisation used: if the neighbourhood of that point is treated as a small pure screw segment then cross slip will be allowed, but if there is a small edge component then cross slip will not occur. This problem is typically solved in discrete dislocation dynamics codes by allowing segments that are within 10-15 degrees of the screw orientation to cross slip.

To avoid this situation in which the numerical discretisation is used to regularise a singular law, we instead regularise the law mathematically. Effectively we are saying that cross slip is possible providing the dislocation is sufficiently close to a pure screw, rather than requiring the dislocation to be exactly pure screw. Specifically we introduce a weighting function $\varphi$, such that

$$
\varphi(0)=1 ; \quad 0 \leq \varphi(x) \leq 1 ; \quad \varphi^{\prime}(x)<0 ; \quad \lim _{x \rightarrow \infty} \varphi=0
$$

and write

$$
\begin{aligned}
\mathbf{M} & =m_{g} \mathbf{I}+m_{g}\left(\varphi\left(\frac{b^{2}-b_{l}^{2}}{b^{2} \epsilon^{2}}\right)-1\right) \boldsymbol{\beta} \otimes \boldsymbol{\beta}, \\
& =m_{g} \mathbf{I}+m_{g}\left(\varphi\left(\frac{\sin ^{2} \alpha}{\epsilon^{2}}\right)-1\right) \boldsymbol{\beta} \otimes \boldsymbol{\beta},
\end{aligned}
$$

where $b_{l}$ is the screw component of the dislocation Burgers vector and $\alpha$ is the tilt angle (the angle between the Burgers vector and the tangent). Here $\epsilon \ll 1$ can be thought as an angle measuring how close to screw a dislocation needs to be in order to cross slip. The simple practical numerical regularisation of allowing cross slip for tilt angles less than 10 degrees corresponds to choosing

$$
\phi(x)= \begin{cases}1 & \text { if } x<1 \\ 0 & \text { if } x \geq 1\end{cases}
$$


and $\epsilon=\sin 10^{\circ} \approx 0.17$. For our mathematical analysis (and it makes more sense physically) we prefer to use a smooth function $\phi$. For the analysis of the instability we will keep $\varphi$ unspecified, so that we can see how the results depend on $\varphi$. We will find that qualitatively our results are independent of the particular choice of $\varphi$, though there is a small quantitative dependence (see, for example, equation (36)). For the numerical results and plots we will choose the simple representative function $\varphi(x)=e^{-x}$. Note that we have taken $m_{c}=0$ for simplicity, but that $\varphi$ can easily be modified to allow for the climb of edge dislocations.

\subsection{Nondimensionalisation}

To calculate the self-induced law of motion for a dislocation curve we need to calculate the self-induced force. In the following section we find the local self-induced stress in the vicinity of an arbitrary curved dislocation through an asymptotic expansion of the Peach-Koehler expression (1). To facilitate the analysis we first nondimensionalise. Although the Burgers vector would seem to be the natural lengthscale, in fact for a curved dislocation the relevant lengthscale is the scale over which this curvature takes place (the inverse of a typical radius of curvature, or the wavelength of small amplitude oscillations). Thus we scale length with a reference length $L$, leaving the definition of $L$ unspecified at this stage. We scale $\boldsymbol{\sigma}$ with $\mu b / 4 \pi L$, and write $\mathbf{b}=b \hat{\mathbf{b}}$ with $\hat{\mathbf{b}}$ of unit modulus. With reference to (4) we scale the force per unit length $\mathbf{f}$ with $\mu b^{2} / 4 \pi L$, and then with reference to (6) we scale time with $4 \pi L^{2} / m_{g} \mu b^{2}$.

\subsection{The local stress field of a curvilinear dislocation}

From the fact that

$$
\nabla Z=\frac{\mathbf{z}}{Z}
$$

the Peach-Koehler stress tensor (1)-(3) can be rewritten in nondimensional form as

$$
\boldsymbol{\sigma}^{\mathbf{1}}=-\int_{C} \frac{\hat{\mathbf{b}} \wedge \mathbf{z}}{Z^{3}} \otimes \mathrm{d} \mathbf{q}, \quad \boldsymbol{\sigma}^{2}=\frac{1}{1-\nu} \int_{C}(\mathrm{~d} \mathbf{q} \cdot(\hat{\mathbf{b}} \wedge \nabla))\left((\nabla \otimes \nabla) Z-\frac{2}{Z} \mathbf{I}\right) .
$$

In order to calculate the self-induced motion of a curved dislocation we need to be able to evaluate the self stress. It is well known that the stress field diverges as $1 /|\mathbf{x}-\mathbf{q}|$ as the point $\mathbf{x}$ approaches a point $\mathbf{q}$ on the dislocation line. Cai et al. regularise the integrals (10) by "smearing out" the Burgers vector over the core region, turning the line integrals into volume integrals [11]. The distribution function of the smeared Burgers vector can even be related to atomistic simulations. They can then evaluate analytically the regularised stress for rectilinear dislocations.

However, we are interested here in curved dislocations, for which such explicit formulae are not available ${ }^{2}$. We are also interested in the limit in which the core region is small by

\footnotetext{
${ }^{2}$ During revision of this work we became aware of the related work by Zhao et al. [32].
} 


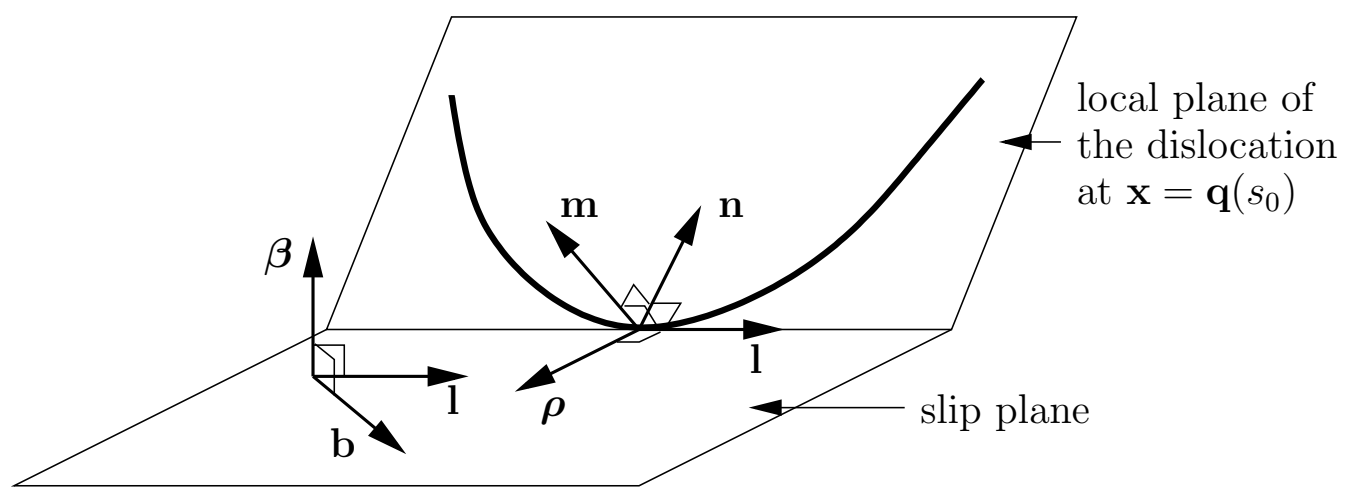

Figure 1: Schematic diagram showing the local orthogonal vectors $\mathbf{1}, \mathbf{n}$ and $\mathbf{m}$, the normal to the slip plane $\boldsymbol{\beta}$, and the normal to the dislocation in the slip plane $\boldsymbol{\rho}$

comparison to the radius of curvature of the dislocation line. In such a limit we can proceed via the method of matched asymptotic expansions. Outside the core region equations (10) hold, and these must be matched with an inner core region in which some regularised model holds, which may be the smeared Burgers vector model of Cai et al., or may be full atomistic simulations.

The integrand in (10) is sufficiently singular that as the point $\mathbf{x}$ approaches the dislocation the dominant contribution to the stress arises from the integral over nearby points on the dislocation curve. We introduce a local curvilinear coordinate system $(s, r, \theta)$ by writing

$$
\mathbf{x}=\mathbf{q}(s)+\mathbf{n}(s) r \cos \theta+\mathbf{m}(s) r \sin \theta,
$$

where $\mathbf{q}(s)$ is the nearest point to $\mathbf{x}$ on the dislocation curve (parameterised by the arc length $s), r$ is the distance from $\mathbf{x}$ to the dislocation, $\mathbf{n}$ is the unit normal to the dislocation curve, and $\mathbf{m}$ is the unit binormal. The vectors $(\mathbf{l}, \mathbf{n}, \mathbf{m})$ form an orthogonal triad, and $r$ and $\theta$ are local polar coordinates in the $\mathbf{n}-\mathbf{m}$ plane (see Figure 1).

We wish to evaluate the stress $\boldsymbol{\sigma}$ as $\mathbf{x}$ approaches a point on the dislocation curve $\mathbf{q}\left(s_{0}\right)$, that is, as $s \rightarrow s_{0}$ and $r \rightarrow 0$. To this end we rescale $r=\varepsilon R$ and consider the limit $\varepsilon \rightarrow 0$. We split the dislocation curve into a local part and non-local part by writing

$$
\int_{C}=\int_{s_{0}-S}^{s_{0}+S}+\int_{C_{1}},
$$

where $S$ is such that $1 /|\log \varepsilon| \ll S \ll 1$. In the local integral the dislocation curve $\mathbf{q}$ can be expanded in the vicinity of $s_{0}$ as

$$
\mathbf{q}(s) \sim \mathbf{q}_{0}+\varepsilon l \mathbf{l}_{0}+\frac{\varepsilon^{2} \kappa_{0} l^{2}}{2} \mathbf{n}_{0}+\cdots,
$$

where $\varepsilon l=s-s_{0}$ is the arc length, $\kappa_{0}$ is the curvature at $s_{0}$, and $\mathbf{q}_{0}=\mathbf{q}\left(s_{0}\right)$, etc. The terms in the integrand can be similarly expanded, such as

$$
\mathbf{z}=\mathbf{q}\left(s_{0}+\varepsilon l\right)-\mathbf{x} \sim-\varepsilon\left(\mathbf{n}_{0} R \cos \theta+\mathbf{m}_{0} R \sin \theta-\mathbf{l}_{0} l\right)+\varepsilon^{2} \mathbf{n}_{0} \frac{\kappa_{0}}{2}+\cdots,
$$


and

$$
\mathrm{d} \mathbf{q}\left(s_{0}+\varepsilon l\right) \sim\left(\varepsilon \mathbf{l}_{0}+\varepsilon^{2} \mathbf{n}_{0} \kappa_{0} l+\mathcal{O}\left(\varepsilon^{3}\right)\right) \mathrm{d} l .
$$

We also write the unit Burgers vector in terms of the local coordinate system at $s_{0}$ as

$$
\hat{\mathbf{b}}=\hat{b}_{n} \mathbf{n}_{0}+\hat{b}_{m} \mathbf{m}_{0}+\hat{b}_{l} \mathbf{l}_{0} .
$$

Henceforth, for ease of notation and without chance of confusion, we drop the subscript 0 from $\mathbf{q}, \mathbf{l}, \mathbf{n}, \mathbf{m}$, and $\kappa$. Now we can incorporate (11), (12), and (13) into (10), and express the result in terms of the local coordinate system $(\mathbf{n}, \mathbf{m}, \mathbf{l})$.

The resulting expansion of $\boldsymbol{\sigma}^{\mathbf{1}}$ and $\boldsymbol{\sigma}^{\mathbf{2}}$ is derived in Appendix 6.1. Combining (37) and (42) we obtain the local expansion for the Peach-Koehler stress tensor in the local coordinate system $(\mathbf{n}, \mathbf{m}, \mathbf{l})$ as

$$
\begin{gathered}
\boldsymbol{\sigma} \sim \frac{2}{r}\left(\begin{array}{ccc}
\frac{-\hat{b}_{n} \sin \theta\left(1+2 \cos ^{2} \theta\right)+\hat{b}_{m} \cos \theta \cos 2 \theta}{1-\nu} & \frac{\hat{b}_{n} \cos \theta \cos 2 \theta+\hat{b}_{m} \sin \theta \cos 2 \theta}{1-\nu} & -\hat{b}_{l} \sin \theta \\
\frac{\hat{b}_{n} \cos \theta \cos 2 \theta+\hat{b}_{m} \sin \theta \cos 2 \theta}{1-\nu} & \frac{\hat{b}_{n} \sin \theta \cos 2 \theta+\hat{b}_{m} \cos \theta\left(1+2 \sin ^{2} \theta\right)}{1-\nu} & \hat{b}_{l} \cos \theta \\
-\hat{b}_{l} \sin \theta & \hat{b}_{l} \cos \theta & -\frac{2 \nu\left(\hat{b}_{n} \sin \theta-\hat{b}_{m} \cos \theta\right)}{1-\nu}
\end{array}\right) \\
+\log \left(\frac{1}{r}\right) \cdot \kappa\left(\begin{array}{ccc}
-\frac{\hat{b}_{m}(1-4 \nu)}{1-\nu} & \frac{\hat{b}_{n}(1-2 \nu)}{1-\nu} & 0 \\
\frac{\hat{b}_{n}(1-2 \nu)}{1-\nu} & \frac{\hat{b}_{m}}{1-\nu} & \frac{\hat{b}_{l}(1+\nu)}{1-\nu} \\
0 & \frac{\hat{b}_{l}(1+\nu)}{1-\nu} & \frac{2 \hat{b}_{m}(5-\nu)}{1-\nu}
\end{array}\right)+\cdots \\
\end{gathered}
$$

as $r \rightarrow 0$. The first term in (14) corresponds to the stress field induced by a rectilinear dislocation. The effect of the curvature of the dislocation is felt in the second term, which is proportional to $\kappa$.

\subsection{Motion of a curvilinear dislocation}

To determine the self-induced motion of a curvilinear dislocation we need to evaluate the local force given by (the nondimensional version of) (4) on the dislocation curve, that is, at $r=0$. We see that the singular nature of the stress causes a problem: the force evaluates to infinity. This singularity is regularised by the finite size of the dislocation core: over length of a few Burgers vectors the Volterra model of a dislocation breaks down, and an alternative (non-singular) model must be used. This could be an atomistic model, or a "smeared" Burgers vector model such as that used by [11].

When the local model is matched to the core model a self-induced law of motion can be determined, as happens in similar scenarios in the field of fluid or superconducting vortex dynamics. Following the systematic procedure of matched inner and outer asymptotic expansions for an atomistic core is very difficult, due to the discrete nature of the inner core model. The "smeared" Burgers vector model of the core region is easier. In this case we will find the first term in the expansion (14) does not generate any self-induced force: rectilinear 
dislocations in an unbounded material have no self-induced motion. The second term will produce a self-induced force

$$
\mathbf{f}_{\mathrm{loc}}=(\hat{\mathbf{b}} \cdot \boldsymbol{\sigma}) \wedge \mathbf{l}=\log \left(\frac{1}{a}\right) \frac{\kappa}{1-\nu}\left(\begin{array}{c}
\hat{b}_{n}^{2}(1-2 \nu)+\hat{b}_{m}^{2}+\hat{b}_{l}^{2}(1+\nu) \\
-2 \hat{b}_{n} \hat{b}_{m} \nu \\
0
\end{array}\right)+\cdots
$$

where $a$ is the (nondimensional) dislocation core radius. This term is independent of the details of the core region, depending only on its radius (and we would expect it to be the same whatever the core model). The particular core model would affect the next term in the expansion, at which order the force is no longer local but depends on the whole dislocation curve. We note that (15) agrees with (and generalises) the expressions for the self-force on circular dislocation loops in [11]. Typically the core radius is taken to be between $b$ and $4 b$, so that $a \approx b / L[20]$.

An alternative way to approach the self-force is to calculate the energy of the dislocation and then to determine the self-force as the variation of this energy. There is still the problem of the singularity, which leads to a formally infinite self-energy. In this case the regularisation is usually performed by defining the self-energy to be the strain energy exterior to a tube of radius a surrounding the dislocation. Such a procedure was carried out by Barnett and Gavazza [16] for planar dislocation loops. The expression (15) agrees with their expressions for circular edge and shear loops, and generalises their results to non-planar dislocations (though, unlike [16], (15) is limited to isotropic materials).

The projection of the unit Burgers vector onto the plane normal to the dislocation, $\hat{\mathbf{b}}-\hat{b}_{l} \mathbf{l}$, plays a key role in the law of motion. Providing the dislocation is not pure screw we can define the unit vector in this direction as

$$
\rho=\frac{\hat{\mathbf{b}}-\hat{b}_{l} \mathbf{l}}{\left|\hat{\mathbf{b}}-\hat{b}_{l} \mathbf{l}\right|}
$$

$\boldsymbol{\rho}$ is the direction normal to the dislocation in the glide plane. By replacing $\hat{b}_{m} \mathbf{m}$ by $\sqrt{1-\hat{b}_{l}^{2}} \boldsymbol{\rho}-\hat{b}_{n} \mathbf{n}$ the local force can be decomposed into components parallel to $\boldsymbol{\rho}$ and n as

$$
\mathbf{f}_{\mathrm{loc}}=\frac{\kappa}{(1-\nu)}\left(\left(1+\nu \hat{b}_{l}^{2}\right) \mathbf{n}-2 \nu \hat{b}_{n} \sqrt{1-\hat{b}_{l}^{2}} \boldsymbol{\rho}\right) \log \left(\frac{1}{a}\right)+\mathcal{O}(1) .
$$

We now consider that in addition to the self-induced force the dislocation is subject to a uniform (nondimensional) external stress $\boldsymbol{\sigma}_{\text {ext }}$.

The presence of $\log (1 / a)$ in $(16)$, with $a \ll 1$, means that the motion of the dislocation is fast on the timescale we have chosen. We may remove this factor from the law of motion by rescaling time with $1 / \log (1 / a)$. After substituting (16) into (6) and using (9) the law of 


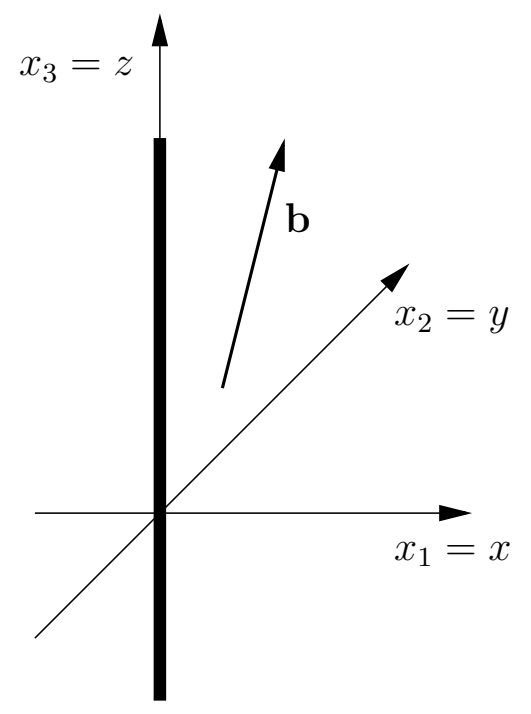

Figure 2: A uniformly translating rectilinear dislocation with tangent $\mathbf{l}=(0,0,1)$ and Burger's vector $\mathbf{b}=b\left(\hat{b}_{1}, 0, \hat{b}_{3}\right)$.

motion is then

$$
\begin{aligned}
\mathbf{v}= & \frac{\varphi\left(\frac{1-\hat{b}_{l}^{2}}{\epsilon^{2}}\right)\left(1+\nu \hat{b}_{l}^{2}\right)}{1-\nu} \kappa \mathbf{n} \\
& -\frac{2 \nu \kappa \hat{b}_{n} \sqrt{1-\hat{b}_{l}^{2}}}{1-\nu} \boldsymbol{\rho}+\frac{\kappa \hat{b}_{n}\left(1-\varphi\left(\frac{1-\hat{b}_{l}^{2}}{\epsilon^{2}}\right)\right)\left(1+\nu \hat{b}_{l}^{2}\right)}{(1-\nu) \sqrt{1-\hat{b}_{l}^{2}}} \boldsymbol{\rho} \\
& +\left(1-\varphi\left(\frac{1-\hat{b}_{l}^{2}}{\epsilon^{2}}\right)\right)(\boldsymbol{\rho} \cdot(\mathbf{F} \wedge \mathbf{l})) \boldsymbol{\rho}+\varphi\left(\frac{1-\hat{b}_{l}^{2}}{\epsilon^{2}}\right)(\mathbf{F} \wedge \mathbf{l}),
\end{aligned}
$$

where $\mathbf{F}=(1 / \log (1 / a)) \boldsymbol{\sigma}_{\text {ext }} \cdot \hat{\mathbf{b}}$ (which we assume is $O(1)$ as $a \rightarrow 0$; this is the distinguished limit). Note that we could add an arbitrary multiple of $\mathbf{l}$ to the right-hand side of (17): such a term does not change the curve $C$, it merely corresponds to a different parametrisation of it.

\section{Linear Stability Analysis}

We consider the stability of a uniformly translating rectilinear dislocation.

Without loss of generality, we choose the $x_{3}$ coordinate to be in the direction of the tangent (see Figure 2). We still have one degree of freedom in orientating the coordinate system. We fix this by choosing the $x_{1}$ direction to be the projection of the Burgers vector in the plane normal to the dislocation, so that $\hat{b}_{1} \geq 0, \hat{b}_{2}=0, \hat{b}_{3} \geq 0$. To simplify the notation slightly 


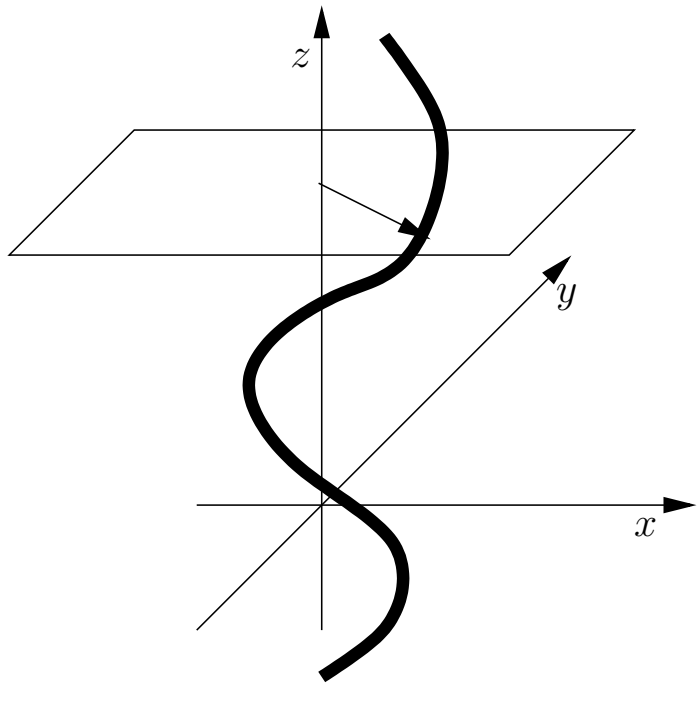

(a)

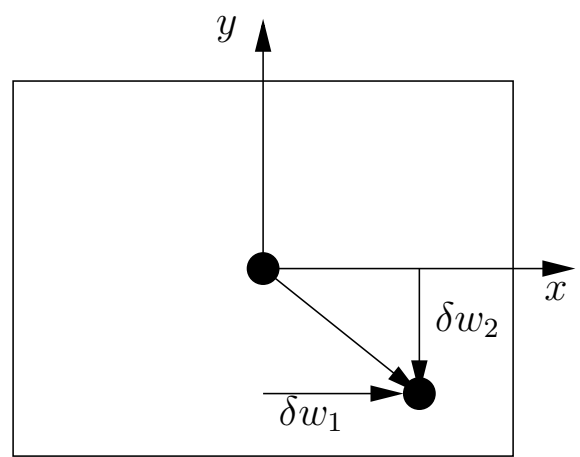

(b)

Figure 3: A perturbed dislocation (a) three dimensional view; (b) in the cross-section $z=$ constant. At each height $z, \delta w_{1}(z)$ is the displacement of the dislocation line in the $x$ direction, and $\delta w_{2}(z)$ is the displacement of the dislocation line in the $y$ direction.

we will hemceforth use $x, y$ and $z$ to denote $x_{1}, x_{2}$ and $x_{3}$ respectively. Then the solution of (17) corresponding to a uniformly translating rectilinear dislocation is

$$
\mathbf{x}_{0}=\left(\begin{array}{c}
F_{2} t \\
-\varphi\left(\frac{1-\hat{b}_{l}^{2}}{\epsilon^{2}}\right) F_{1} t \\
z
\end{array}\right) .
$$

For $\hat{b}_{1}>0$, we perturb this solution by setting

$$
\mathbf{x}=\mathbf{x}_{0}+\delta\left(\begin{array}{c}
w_{1} \\
w_{2} \\
0
\end{array}\right)
$$

where $\delta \ll \epsilon^{2}$. At each height $z, \delta w_{1}(z)$ is the displacement of the dislocation line in the $x$ direction, and $\delta w_{2}(z)$ is the displacement of the dislocation line in the $y$ direction (see Figure 3). Then

$$
\begin{gathered}
\mathbf{l} \sim\left(\begin{array}{l}
0 \\
0 \\
1
\end{array}\right)+\delta\left(\begin{array}{c}
w_{1, z} \\
w_{2, z} \\
0
\end{array}\right) ; \quad \kappa \mathbf{n} \sim \delta\left(\begin{array}{c}
w_{1, z z} \\
w_{2, z z} \\
0
\end{array}\right) ; \quad \mathbf{n} \sim \frac{1}{\sqrt{w_{1, z z}^{2}+w_{2, z z}^{2}}}\left(\begin{array}{c}
w_{1, z z} \\
w_{2, z z} \\
0
\end{array}\right) ; \\
\boldsymbol{\rho} \cdot(\mathbf{F} \wedge \mathbf{l}) \sim F_{2}+\delta \frac{F_{1} \hat{b}_{3}-F_{3} \hat{b}_{1}}{\hat{b}_{1}} w_{2, z} ; \quad \varphi\left(\frac{1-\hat{b}_{l}^{2}}{\epsilon^{2}}\right) \sim \varphi\left(\frac{\hat{b}_{1}^{2}}{\epsilon^{2}}\right)-2 \delta w_{1, z} \frac{\hat{b}_{1} \hat{b}_{3}}{\epsilon^{2}} \varphi^{\prime}\left(\frac{\hat{b}_{1}^{2}}{\epsilon^{2}}\right) .
\end{gathered}
$$


Collecting the $\mathcal{O}(\delta)$ terms in the law of motion (17) gives the evolution of the perturbation as

$$
\begin{aligned}
w_{1, t}= & \frac{1}{1-\nu} \cdot\left(\hat{b}_{3}^{2}(1+\nu)+\hat{b}_{1}^{2}(1-2 \nu)\right) w_{1, z z}-\left((\varphi-1) F_{1} \hat{b}_{3} / \hat{b}_{1}+F_{3}\right) w_{2, z}, \\
w_{2, t}= & \frac{\varphi}{1-\nu} \cdot\left(\hat{b}_{3}^{2}(1+\nu)+\hat{b}_{1}^{2}\right) w_{2, z z} \\
& \quad+\varphi F_{3} w_{1, z}+\frac{2 \varphi^{\prime} \hat{b}_{1} \hat{b}_{3}}{\epsilon^{2}} F_{1} w_{1, z}+(\varphi-1) \frac{F_{2} \hat{b}_{3}}{\hat{b}_{1}} w_{2, z},
\end{aligned}
$$

where $\varphi$ and $\varphi^{\prime}$ denote $\varphi\left(\hat{b}_{1}^{2} / \epsilon^{2}\right)$ and $\varphi^{\prime}\left(\hat{b}_{1}^{2} / \epsilon^{2}\right)$, respectively.

We look for solutions of the form

$$
w_{1}=A_{1} e^{\lambda t} \cos k z+B_{1} e^{\lambda t} \sin k z, \quad w_{2}=A_{2} e^{\lambda t} \cos k z+B_{2} e^{\lambda t} \sin k z
$$

We find that (21) satisfies (19)-(20) if and only if

$$
\left(\begin{array}{cccc}
\lambda+c_{1} k^{2} & 0 & 0 & -a_{12} k \\
0 & a_{12} k & \lambda+c_{1} k^{2} & 0 \\
0 & \lambda+c_{2} k^{2} & -a_{21} k & -a_{22} k \\
a_{21} k & a_{22} k & 0 & \lambda+c_{2} k^{2}
\end{array}\right)\left(\begin{array}{c}
A_{1} \\
A_{2} \\
B_{1} \\
B_{2}
\end{array}\right)=\left(\begin{array}{l}
0 \\
0 \\
0 \\
0
\end{array}\right)
$$

where

$$
\begin{gathered}
c_{1}=\frac{1}{1-\nu} \cdot\left(\hat{b}_{3}^{2}(1+\nu)+\hat{b}_{1}^{2}(1-2 \nu)\right), \quad c_{2}=\frac{\varphi}{1-\nu} \cdot\left(\hat{b}_{3}^{2}(1+\nu)+\hat{b}_{1}^{2}\right) ; \\
a_{12}=-\left((\varphi-1) F_{1} \hat{b}_{3} / \hat{b}_{1}+F_{3}\right), \quad a_{21}=\varphi F_{3} w_{1, z}+\frac{2 \varphi^{\prime} \hat{b}_{1} \hat{b}_{3}}{\epsilon^{2}} F_{1}, \quad a_{22}=(\varphi-1) \frac{F_{2} \hat{b}_{3}}{\hat{b}_{1}} .
\end{gathered}
$$

Note that, since $\nu<1 / 2, c_{1}$ and $c_{2}$ are positive. A non-zero solution exists if and only if the determinant of the matrix vanishes, which gives the dispersion relation between $\lambda$ and $k$. The cumbersome calculations are presented in Appendix 6.2, with the result that

$$
\begin{aligned}
\Re(\lambda)= & -\frac{\left(c_{1}+c_{2}\right) k^{2}}{2} \\
& \pm \frac{k}{2 \sqrt{2}}\left(\left(\left(c_{1}-c_{2}\right)^{4} k^{4}+2\left(c_{1}-c_{2}\right)^{2}\left(a_{22}^{2}-4 a_{12} a_{21}\right) k^{2}+\left(a_{22}^{2}+4 a_{12} a_{21}\right)^{2}\right)^{1 / 2}\right. \\
& \left.\quad+\left(c_{1}-c_{2}\right)^{2} k^{2}-\left(a_{22}^{2}+4 a_{12} a_{21}\right)\right)^{1 / 2} \cdot
\end{aligned}
$$

The dependence of $\lambda$ in (25) on the direction of the Burgers vector and the applied stresses (through (23) and (24)) is difficult to interpret in general. To tease out this information we consider the limit in which $\epsilon \rightarrow 0$, that is, in which only dislocations which are close to screw can cross slip. The behaviour of the equations then depends crucially on how close to a pure screw the original dislocation is, that is, it depends on the size of $\hat{b}_{1}$. We consider each distinguished limit in turn. 


\subsection{Perturbation to a mixed dislocation $\left(\hat{b}_{1}=\mathcal{O}(1)\right)$}

In this regime, $\varphi\left(\hat{b}_{1}^{2} / \epsilon^{2}\right) \ll 1$, and $(25)$ simplifies to

$$
\Re(\lambda) \sim-c_{1} k^{2}, \quad \Re(\lambda) \sim 0 .
$$

The origin of these eigenvalues can be seen by observing that in this limit (19) and (20) can be reduced to

$$
\begin{aligned}
& w_{1, t}=\frac{1}{1-\nu}\left(\hat{b}_{3}^{2}(1+\nu)+\hat{b}_{1}^{2}(1-2 \nu)\right) w_{1, z z}-\left(F_{3}-F_{1} \frac{\hat{b}_{3}}{\hat{b}_{1}}\right) w_{2, z}, \\
& w_{2, t}=-\frac{F_{2} \hat{b}_{3}}{\hat{b}_{1}} w_{2, z}
\end{aligned}
$$

at leading order in $\epsilon$. The first (stable) eigenvalue corresponds to the line tension in a planar dislocation line. The second (neutrally stable) eigenvalue corresponds to a local change in the slip plane for line segments which are perturbed out of the $(x, z)$-plane. In this regime the dislocation line is therefore stable.

\subsection{Perturbation to an almost-screw dislocation $\left(\hat{b}_{1}=\mathcal{O}(\epsilon)\right)$}

We set $\hat{b}_{1}=\bar{b}_{1} \epsilon$. Then $\hat{b}_{1}^{2} / \epsilon^{2}=\bar{b}_{1}^{2}$ is $\mathcal{O}(1)$, so that in this regime $\varphi$ and $\varphi^{\prime}$ denote $\varphi\left(\bar{b}_{1}^{2}\right)$ and $\varphi^{\prime}\left(\bar{b}_{1}^{2}\right)$, respectively. We can see from (26) and (27) that the coefficents of $w_{2, z}$ are becoming large in this regime. This means that the relevant wavenumbers are large. Writing

$$
k=\frac{(1-\nu) \sigma_{13} \hat{k}}{\epsilon(1-\phi)(1+\nu)}, \quad \lambda=\frac{(1-\nu) \sigma_{13}^{2} \hat{\lambda}}{\epsilon^{2}(1+\nu)} .
$$

equation (25) reduces at leading order to

$$
\Re(\hat{\lambda})=\frac{\hat{k}}{2 \sqrt{2}(1-\phi)} \sqrt{\sqrt{\hat{k}^{4}+\hat{L}^{2}+2 \hat{k}^{2} \hat{Q}}+\hat{k}^{2}-\hat{L}}-\frac{(1+\varphi) \hat{k}^{2}}{2(1-\phi)^{2}},
$$

where

$$
\hat{L}=(1-\varphi)^{2} \frac{1}{\bar{b}_{1}^{2}} \frac{\sigma_{23}^{2}}{\sigma_{13}^{2}}+8(1-\varphi) \varphi^{\prime}, \quad \hat{Q}=(1-\varphi)^{2} \frac{1}{\bar{b}_{1}^{2}} \frac{\sigma_{23}^{2}}{\sigma_{13}^{2}}-8(1-\varphi) \varphi^{\prime} .
$$

The remaining parameters are $\bar{b}_{1}$, which measures the edge component of the unit Burgers vector, and the ratio of shear stresses $\sigma_{23} / \sigma_{13}$ (recall that $\phi$ is a function of $\bar{b}_{1}$ ). Fig. 4 shows maximum $\Re(\hat{\lambda})_{\max }$ as a function of $\bar{b}_{1}$ and $\sigma_{23}^{2} / \sigma_{13}^{2}$.

By analysing (28) we find that the dislocation is unstable if

$$
\frac{\sigma_{23}^{2}}{\sigma_{13}^{2}}<-\frac{2 \bar{b}_{1}^{2}(1+\phi)^{2} \phi^{\prime}}{(1-\phi) \phi}
$$




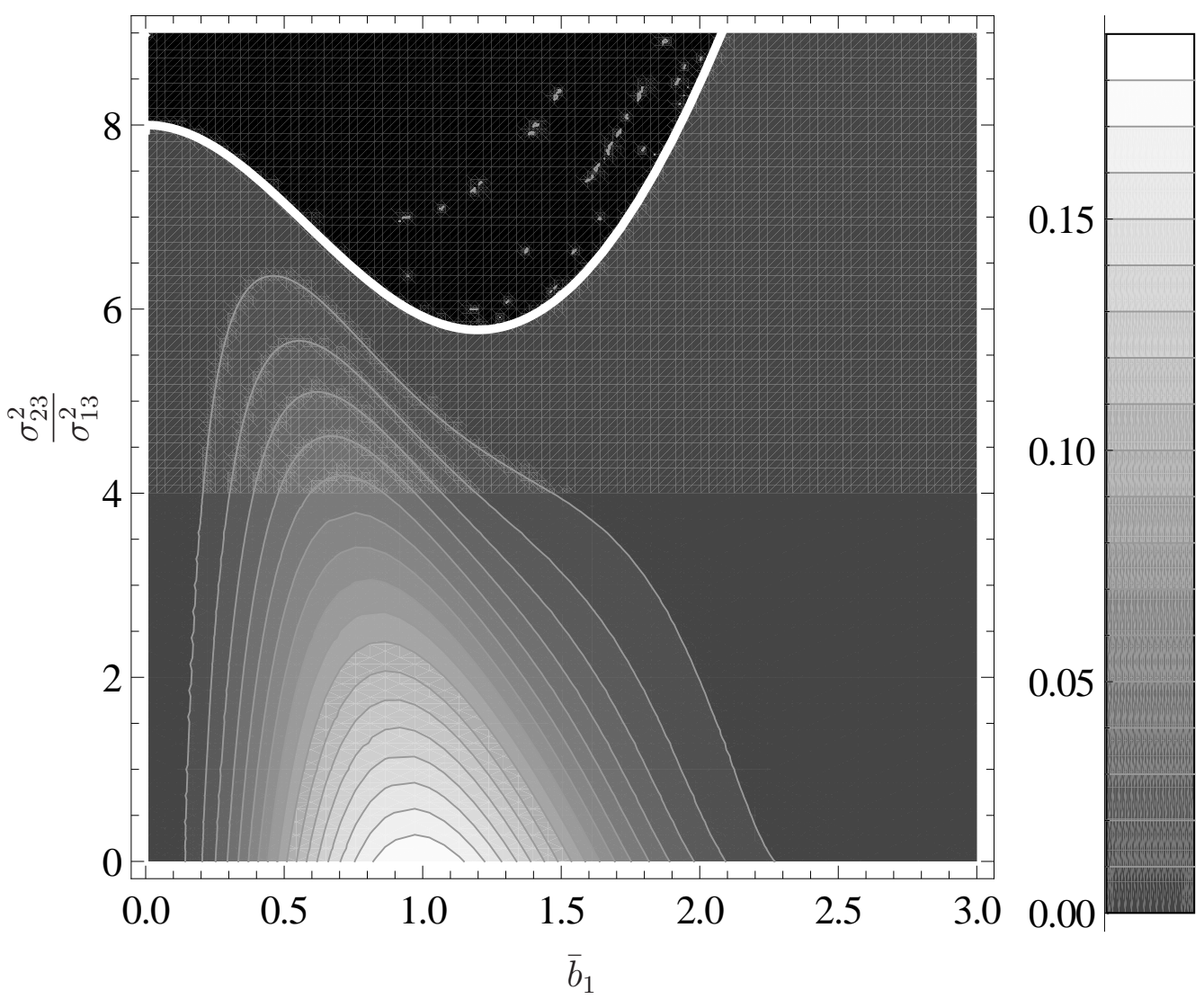

Figure 4: The rescaled maximum growth rate $\Re(\hat{\lambda})_{\max }$ as a function of $\bar{b}_{1}$ and $\sigma_{23}^{2} / \sigma_{13}^{2}$. The dislocation is unstable in the region below the solid white curve. The maximum growth rate is 0.18 and occurs for $\sigma_{23}=0, \bar{b}_{1} \approx 0.981$ with a corresponding wavenumber $\hat{k} \approx 0.54$.

which is shown as the solid line in Figure 4 (remember that $\phi^{\prime}<0$, so the right-hand side of (29) is positive). Note that the unstable region grows as $\bar{b}_{1}$ grows, which seems to contradict the fact that the dislocation is stable for $\hat{b}_{1}$ of order one. However, as $\bar{b}_{1}$ grows the growth rate decays to zero exponentially and the wavenumber also tends zero, so that these (marginally) unstable modes match into the neutrally stable mode of the mixed dislocation.

The most unstable mode occurs when $\sigma_{23}=0, \bar{b}_{1} \approx 0.981$ with a corresponding wavenumber $\hat{k} \approx 0.54$.

\subsection{Perturbation to a screw-dominant dislocation $\left(\hat{b}_{1}=\mathcal{O}\left(\epsilon^{2}\right)\right)$}

There is another distinguished limit when $\hat{b}_{1}=\mathcal{O}\left(\epsilon^{2}\right)$. We set $\hat{b}_{1}=\tilde{b}_{1} \epsilon^{2}$. Then

$$
\hat{b}_{3}=1-\frac{\tilde{b}_{1}^{2}}{2} \epsilon^{4}+o\left(\epsilon^{4}\right), \quad \varphi\left(\frac{\hat{b}_{1}^{2}}{\epsilon^{2}}\right)=\varphi\left(\tilde{b}_{1}^{2} \epsilon^{2}\right)=1+\tilde{b}_{1}^{2} \varphi_{0}^{\prime} \epsilon^{2}+\mathcal{O}\left(\epsilon^{4}\right),
$$


where $\varphi_{0}^{\prime}=\varphi^{\prime}(0)$. Incorporating these into (25) gives at leading order

$$
\Re(\lambda)=-\frac{(1+\nu)}{(1-\nu)} k^{2}+\frac{k}{2 \sqrt{2}} \sqrt{|S|-S},
$$

where

$$
S=\left(\sigma_{23} \phi_{0}^{\prime} \tilde{b}_{1}\right)^{2}-4 \sigma_{33}^{2}-4 \sigma_{13} \sigma_{33} \phi_{0}^{\prime} \tilde{b}_{1}-8\left(\sigma_{13} \phi_{0}^{\prime} \tilde{b}_{1}\right)^{2} .
$$

We see that there are eigenvalues with positive real part if $S<0$, i.e.

$$
\tilde{b}_{1}^{2} \sigma_{23}^{2} \varphi_{0}^{\prime 2}-4\left(\tilde{b}_{1} \sigma_{13} \varphi_{0}^{\prime}+\sigma_{33}\right)\left(2 \tilde{b}_{1} \sigma_{13} \varphi_{0}^{\prime}+\sigma_{33}\right) \geq 0 .
$$

The maximum growth rate and corresponding wavelength are

$$
\begin{aligned}
\Re(\lambda)_{\max } & =\frac{(1-\nu)}{(1+\nu)} \frac{\sigma_{13}^{2}}{16}\left(4\left(\tilde{b}_{1} \varphi_{0}^{\prime}+\frac{\sigma_{33}}{\sigma_{13}}\right)\left(2 \tilde{b}_{1} \varphi_{0}^{\prime}+\frac{\sigma_{33}}{\sigma_{13}}\right)-\tilde{b}_{1}^{2} \frac{\sigma_{23}^{2}}{\sigma_{13}^{2}} \varphi_{0}^{\prime 2}\right), \\
k_{\max } & =\frac{(1-\nu)}{4(1+\nu)} \sqrt{4\left(\tilde{b}_{1} \sigma_{13} \varphi_{0}^{\prime}+\sigma_{33}\right)\left(2 \tilde{b}_{1} \sigma_{13} \varphi_{0}^{\prime}+\sigma_{33}\right)-\tilde{b}_{1}^{2} \sigma_{23}^{2} \varphi_{0}^{\prime 2}} .
\end{aligned}
$$

From (32) and (33) we see that, in this regime, the instability depends on not only on the shear stresses, but also on the normal stress $\sigma_{33}$; the key parameters are $\tilde{b}_{1}, \sigma_{23} / \sigma_{13}$, and $\sigma_{33} / \sigma_{13}$. We see from (32) that for large $\tilde{b}_{1}$ the dislocation is stable if and only if $\sigma_{23} / \sigma_{13} \geq \sqrt{8}$.

In the limit in which $\tilde{b}_{1} \ll 1$, so that the dislocation is even closer to a pure screw, the dispersion relation can be written explicitly as

$$
\Re(\lambda)=-\left(\sigma_{33} k+\frac{(1+\nu) k^{2}}{(1-\nu)}\right),
$$

so that there is a band of unstable wavenumbers

$$
k \in \begin{cases}\left(0,-\frac{(1-\nu) \sigma_{33}}{(1+\nu)}\right), & \sigma_{33}<0 \\ \left(-\frac{(1-\nu) \sigma_{33}}{(1+\nu)}, 0\right), & \sigma_{33}>0\end{cases}
$$

In this regime, the stability and growth rate depend only on the normal stress. We see that the dislocation is always unstable for small $\tilde{b}_{1}$ if $\sigma_{33} \neq 0$.

Fig. 5 depicts the maximum growth rate against $\tilde{b}_{1}$ for various combinations of applied stress. We see that even when the dislocation is unstable for small and large $\tilde{b}_{1}$ there may be a region of stability at moderate $\tilde{b}_{1}$ depending on the relative size and signs of $\sigma_{13}, \sigma_{23}$ and $\sigma_{33}$. 


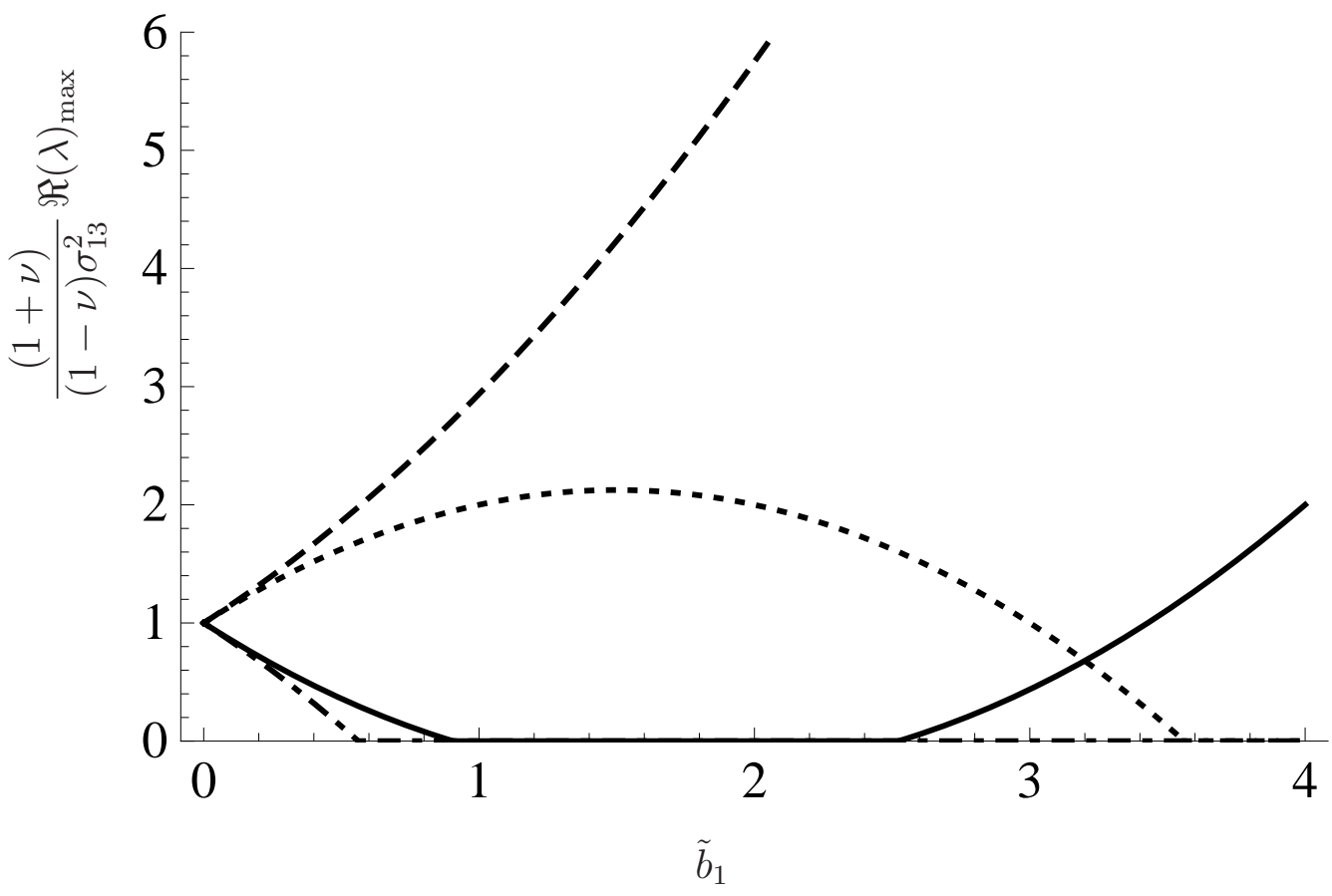

Figure 5: The maximum growth rate as a function of $\tilde{b}_{1}$. The solid curve corresponds to $\sigma_{23} / \sigma_{13}=1, \sigma_{33} / \sigma_{13}=2$; the dashed curve corresponds to $\sigma_{23} / \sigma_{13}=1, \sigma_{33} / \sigma_{13}=-2$; the dotted curve corresponds to $\sigma_{23} / \sigma_{13}=4, \sigma_{33} / \sigma_{13}=-2$; the dot-dashed curve corresponds to $\sigma_{23} / \sigma_{13}=4, \sigma_{33} / \sigma_{13}=2$. The dislocation is always unstable for $\tilde{b}_{1}$ small. For large $\tilde{b}_{1}$ it is stable if and only if $\sigma_{23} / \sigma_{13} \geq \sqrt{8}$. Even when the dislocation is unstable for small and large $\tilde{b}_{1}$ there may be a region of stability at moderate $\tilde{b}_{1}$ depending on the relative size and signs of $\sigma_{13}, \sigma_{23}$ and $\sigma_{33}$.

\subsection{Numerical results}

Having understood how the behaviour of (25) depends on the parameters $b_{1}, \sigma_{11}, \sigma_{23}, \sigma_{33}$ and $\epsilon$ we are now in a position to plot the maximum growth rate as a function of $b_{1}$ for various representative parameter combinations. In fig. 6 we show the maximum growth rate as a function of $\hat{b}_{1}$ on a log-log plot to cope with the vastly different growth rates for small and large $\hat{b}_{1}$.

We see that when the shear-driven instability of $\S 3.2$ is present it dominates, with a growthrate which is orders of magnitude greater than the normal-stress-driven instability of $\S 3.3$.

We may summarise the results of our stability calculations as follows.

(i) When $\hat{b}_{1}=\mathcal{O}(1)$, the dislocation is stable to any planar perturbation.

(ii) When $\hat{b}_{1}=\mathcal{O}(\epsilon)$, the dislocation is unstable if the shear-stress ratio $\sigma_{23} / \sigma_{13}$ is less than a critical value which depends only on $\hat{b}_{1}$. The wavelength of the most unstable mode is small $(\mathcal{O}(\epsilon))$, and the growth rate is large $\left(\mathcal{O}\left(\epsilon^{-2}\right)\right)$.

(iii) When $\hat{b}_{1}=\mathcal{O}\left(\epsilon^{2}\right)$, the dislocation is unstable whenever $\sigma_{33}$ is non zero, for sufficiently 


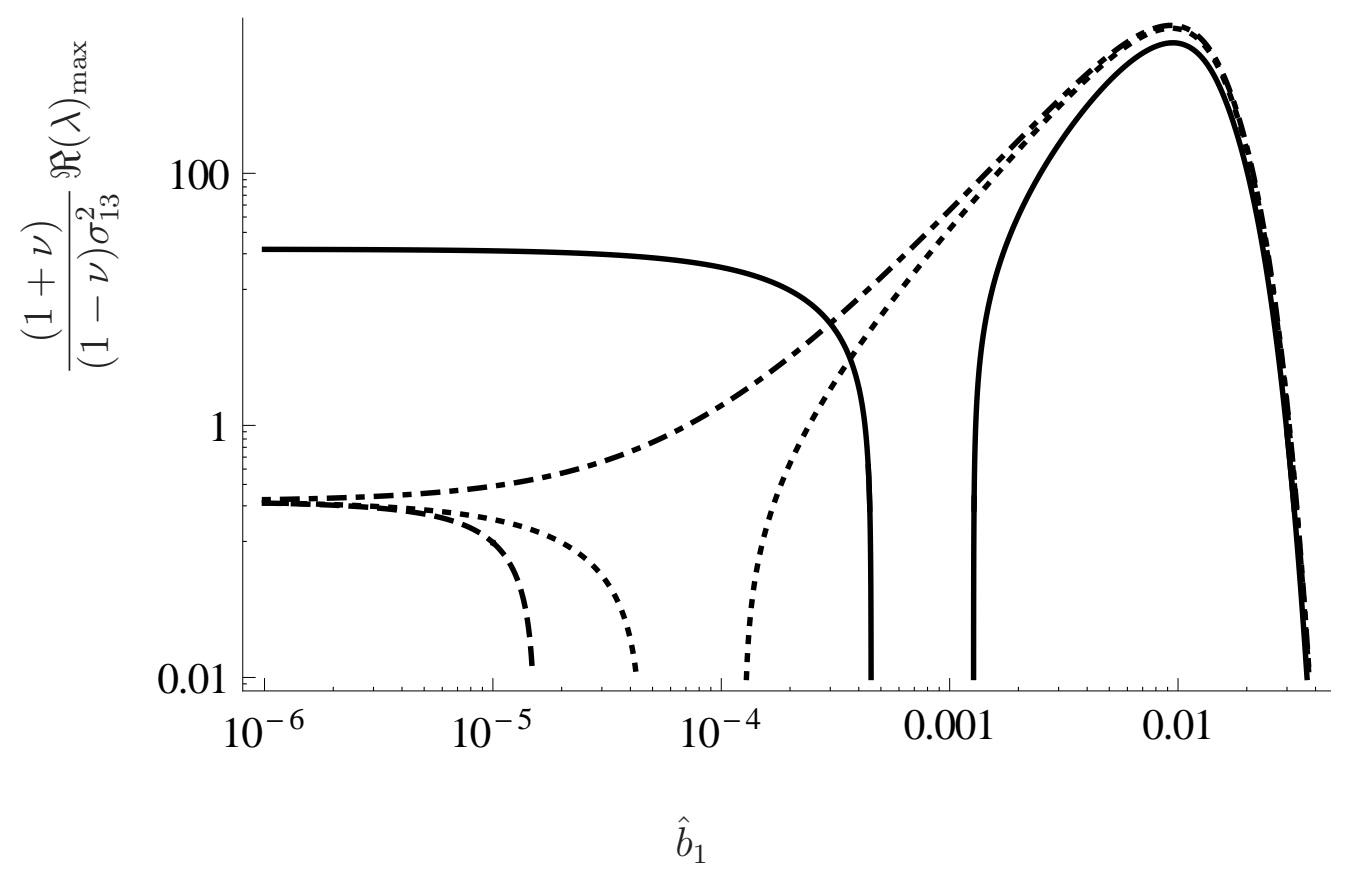

Figure 6: The maximum growth rate as a function of $\hat{b}_{1}$ for various combinations of applied stress. the solid curve corresponds to $\sigma_{23} / \sigma_{13}=1, \sigma_{33} / \sigma_{13}=10$; the dashed curve corresponds to $\sigma_{23} / \sigma_{13}=10, \sigma_{33} / \sigma_{13}=1$; the dotted curve corresponds to $\sigma_{23} / \sigma_{13}=1$, $\sigma_{33} / \sigma_{13}=1$; the dot-dashed curve corresponds to $\sigma_{23} / \sigma_{13}=-1, \sigma_{33} / \sigma_{13}=-1$. For all plots $\epsilon=0.01$.

small $\hat{b}_{1}$. The wavelength and growth rate of this instability are $\mathcal{O}(1)$.

\section{Non-linear evolution of the instability}

The linear stability analysis is useful for determining the stability of a rectilinear dislocation. However, to track the non-linear evolution of the instabilities we have found, we need to solve the evolution equation (17) numerically.

We discretise $\mathbf{q}$ and $\mathbf{v}$ in time and space. At the $n$th timestep we use a TVD Runge-Kunta method described in [21] to compute $\mathbf{q}^{n+1}$ from $\mathbf{q}^{n}$ and $\mathbf{v}^{n}$ via the following scheme:

$$
\begin{gathered}
\mathbf{q}^{n+1}=\mathbf{q}^{n}+\mathbf{v}^{n} \Delta t, \quad \mathbf{q}^{n+2}=\mathbf{q}^{n+1}+\mathbf{v}^{n+1} \Delta t, \quad \mathbf{q}^{n+1 / 2}=\frac{3}{4} \mathbf{q}^{n}+\frac{1}{4} \mathbf{q}^{n+2}, \\
\mathbf{q}^{n+3 / 2}=\mathbf{q}^{n+1 / 2}+\mathbf{v}^{n+1 / 2} \Delta t, \quad \mathbf{q}^{n+1}=\frac{1}{3} \mathbf{q}^{n}+\frac{2}{3} \mathbf{q}^{n+3 / 2}
\end{gathered}
$$

all the other variables are updated in the sequence

$$
\mathbf{q} \Rightarrow \mathbf{l} \Rightarrow \kappa \mathbf{n} \Rightarrow a_{1}, a_{3} \Rightarrow \boldsymbol{\rho} \Rightarrow \mathbf{v}
$$


using the definitions

$$
\mathbf{l}=\frac{\mathbf{q}_{z}}{\left|\mathbf{q}_{z}\right|}, \quad \mathbf{n}=\frac{\mathbf{l}_{z}}{\left|\mathbf{l}_{z}\right|}
$$

The derivatives of $\mathbf{q}$ are approximated by

$$
\left.\frac{\partial \mathbf{q}}{\partial z}\right|_{z=z_{i}}=\frac{\mathbf{q}_{i+1}-\mathbf{q}_{i-1}}{2 \Delta z}
$$

and

$$
\left.\frac{\partial^{2} \mathbf{q}}{\partial z^{2}}\right|_{z=z_{i}}=\frac{\mathbf{q}_{i+1}+\mathbf{q}_{i-1}-2 \mathbf{q}_{i}}{\Delta z^{2}}
$$

at $z_{i}$, where $\Delta z$ is the spatial grid size.

We note the following three points. Firstly, we use the approximation that

$$
\kappa \mathbf{n}=\frac{\partial \mathbf{l}}{\partial z}=\frac{1}{\left|\mathbf{q}_{z}\right|^{2}}\left(\mathbf{q}_{z z}-\left(\mathbf{l} \cdot \mathbf{q}_{z z}\right) \mathbf{q}_{z z}\right)
$$

to keep the truncation error $O\left(\Delta z^{2}\right)$. Secondly, we impose periodic boundary conditions on all variables except the $z$-component of displacement $q_{3}$, for which we impose

$$
q_{3}\left(z_{1}\right)-q_{3}\left(z_{2}\right)=z_{1}-z_{2},
$$

where $z_{1}$ and $z_{2}$ are the two end points. Finally, to avoid calculating the CFL condition at each time step we simply chose $\Delta t=0.1 \Delta z^{2}$, which was enough top give numerical stability for the examples we considered.

\subsection{Numerical results}

We take $\epsilon=0.1$ and $\nu=0.3$.

We begin by perturbing a dislocation in which $\hat{\mathbf{b}} \approx(0.1,0,0.99)^{T}$ and the only nonzero external stress is $\sigma_{13}=4 \pi$. We choose the wavenumber of the initial perturbation to be $k=30$, which is close to that for maximal growth. We discretize space so that there are 100 points per wavelength, which corresponds to $\Delta z=0.0021$. We take $\Delta t=10^{-7}$.

Fig. 7 gives the evolution of the dislocation curve from various angles as well as its projection onto the coordinate planes. Fig. 7(b) depicts its projection to the $x$-y plane, from which the expansion can be seen; Fig. 7 (c) depicts its projection to the $x-z$ plane, from which the glide planes parallel to Burgers vector can be identified; Fig. 7(d) depicts its projection to the $y-z$ plane, from which we can see some parts in the dislocation curve pinned, while the other parts grow.

To try to understand this evolution we show in Fig. 8(a) the proportion of the velocity in the direction normal to the glide plane $\boldsymbol{\beta}$ as a function of the spatial parameter $z$. We see that most parts of the dislocation are moving in the glide plane, with zero velocity in the cross slip direction. Fig. 8(b) shows the proportion of the dislocation which is screw at every 


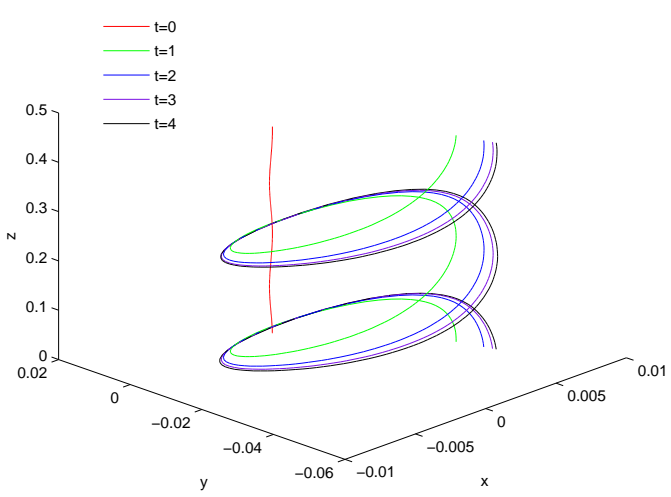

(a)

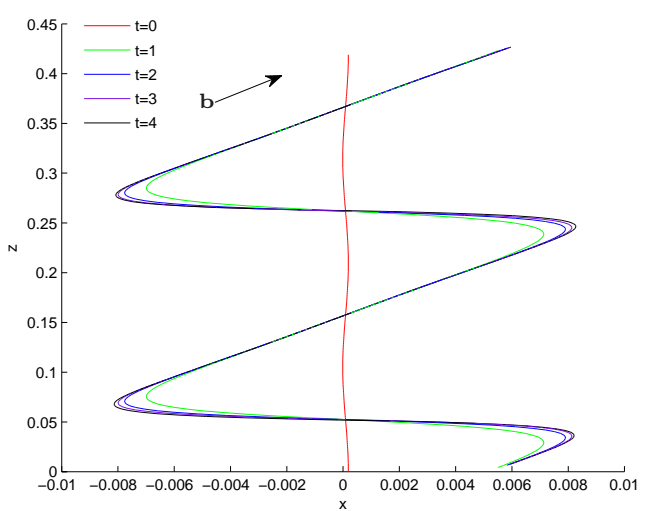

(c)

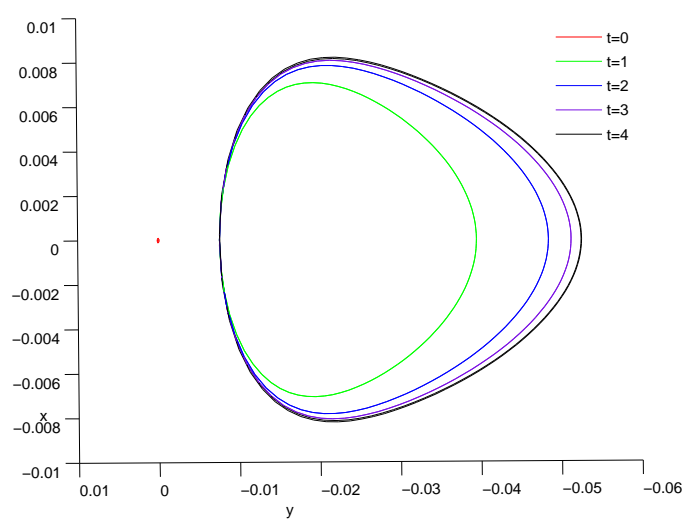

(b)

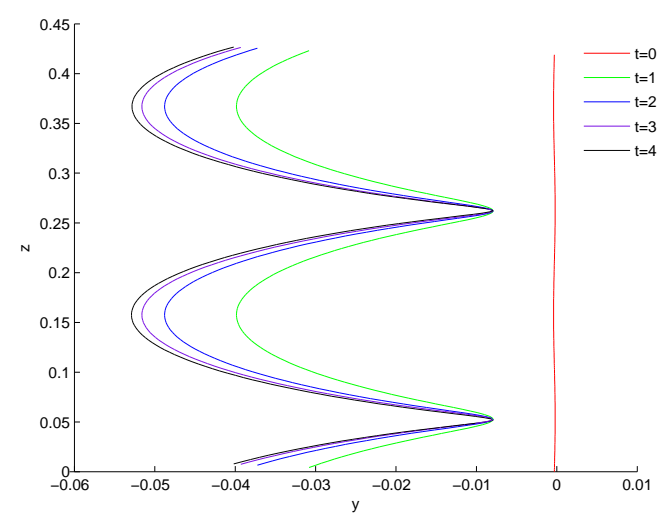

(d)

Figure 7: Non-linear evolution of a perturbed rectilinear dislocation. (a) three-dimensional view; (b) projection onto the $x-y$ plane; (c) projection onto the $x-z$ plane; (d) projection to the $y-z$ plane. The only nonzero component of the (nondimensional) external stress is $\sigma_{13}=4 \pi$.

point, again parameterised by $z$. The parts which are close to pure screw are those which are cross slipping, as we would expect. Noting that initially the dislocation was almost screw, we see that the perturbation has evolved the dislocation into mixed-type undergoing in-plane motion for most of its length.

We can see from Fig $7(\mathrm{~b})$ that for the current parameter values the dislocation will reach a new non-rectilinear equilibrium, in which the "line tension" of the curved parts of the dislocation balances the external stress driving the expansion. This is rather like the way forest interactions may pin a dislocation if the external stress is not too large.

When we increase the only non-vanishing component of the external stress to $\sigma_{13}=20 \pi$, again using an initial perturbation with wavenumber $k=30$, the evolution behaves differently, as shown in Fig. 9 (for the numerical simulations we choose this time 1000 points per wavelength, corresponding to $\Delta z=2.0944 \times 10^{-4}$, and take $\left.\Delta t=5 \times 10^{-9}\right)$. In this case the 


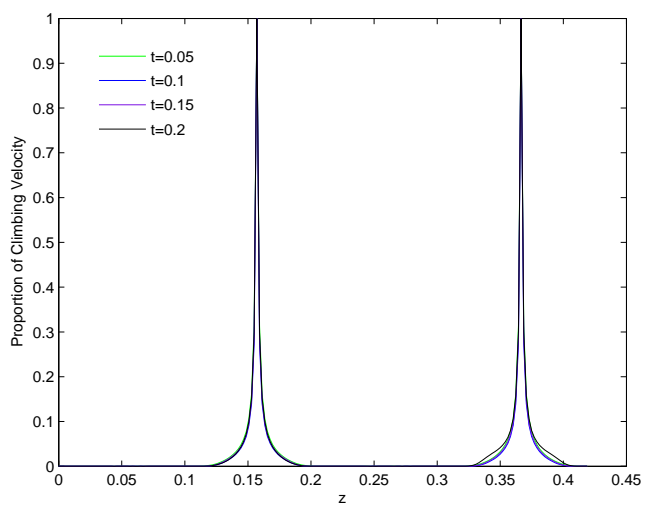

(a)

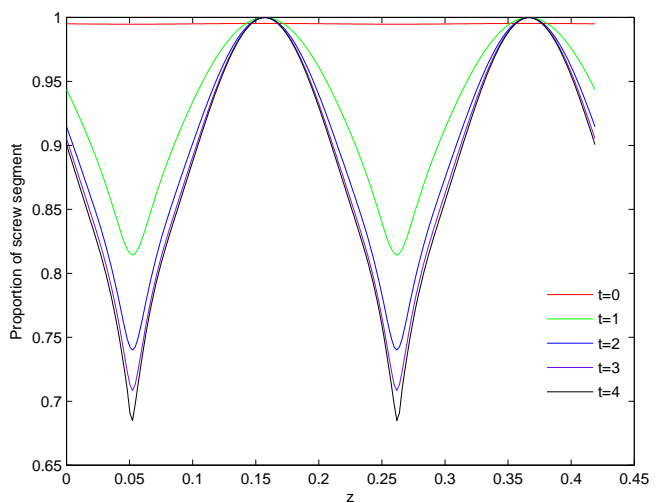

(b)

Figure 8: The left plot gives the proportion of velocity in $\boldsymbol{\beta}$ (normal to the glide plane) direction against the spatial parameter $z$, showing that most parts of the dislocation do in-plane motion. The right plot depicts the proportion of screw segment at every point, parameterised by $z$, showing that the edge components grow when evolving.

external stress is strong enough that no new equilibrium is found.

Fig. 9(a) shows the 3-D plot for the evolution. Fig. 9(b) shows the projection onto the $x-y$ plane, again showing the the expansion due to instability. Fig. 9(c) shows the projection onto the $x-z$ plane; the family of glide planes, which are all perpendicular to $x-z$ plane, can be identified. Fig. 9(d) shows the projection onto the $y$ - $z$ plane; as before, some points of the dislocation are pinned in this projection.

\section{Conclusions}

\subsection{Spacing and orientation of active slip planes}

While Figures 7 and 9 are useful, they are not so easy to interpret. It is of some use to illustrate schematically the non-linear evolution of the dislocation line, as in Fig. 10.

After some time from the initial perturbation the dislocation line will comprise two sets of curves. The expanding blue curves lie in a family of parallel slip planes determined by the applied stress. They are joined by the red segments, which are straight lines lying in a different slip plane (with normal $(0,1,0)$ ), which is the glide plane of the original straight dislocation. Each of these joining red lines rotates around its central point and grows in length as the blue curves expand. Cross slip occurs only at the joins of the blue and red segments. The intersections of the two slip systems are, of course, parallel to the Burgers vector.

The slip planes for the expanding blue segments are those which maximise the resolved shear 


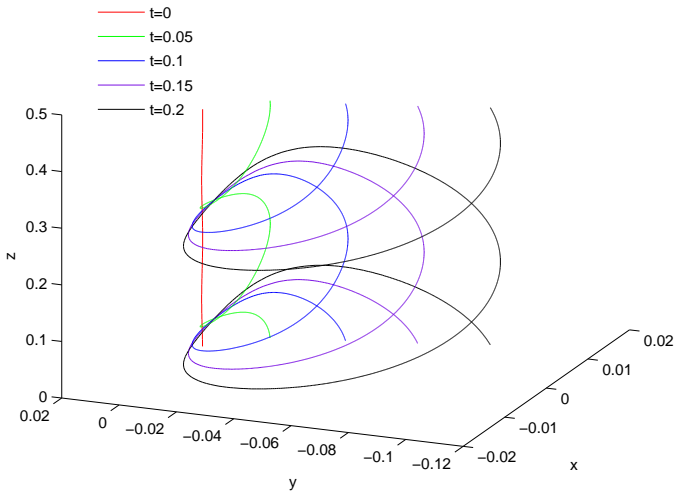

(a)

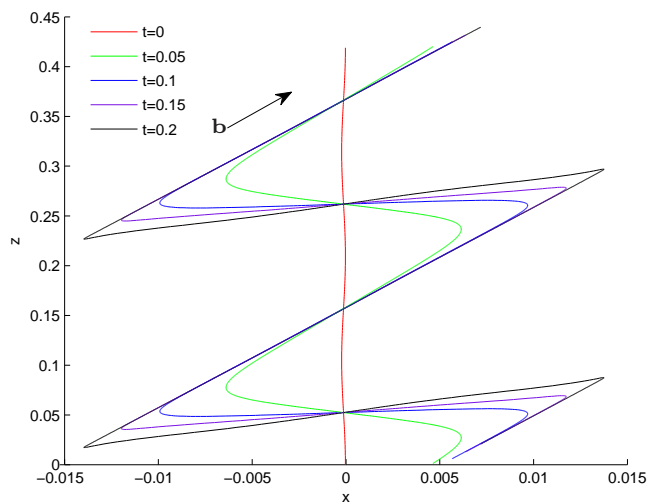

(c)

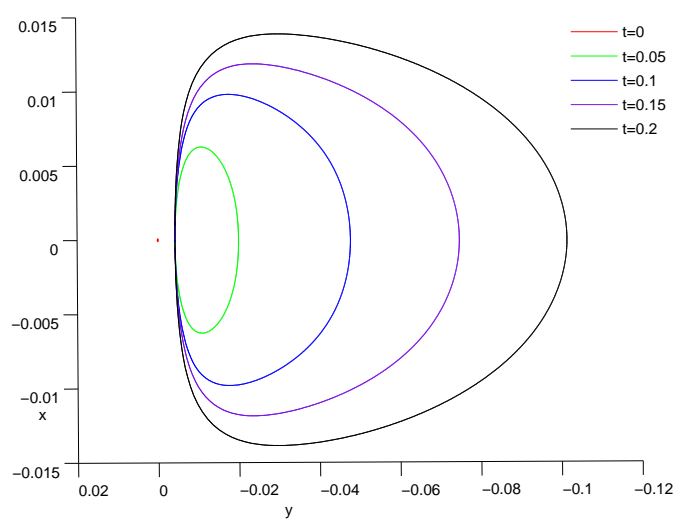

(b)

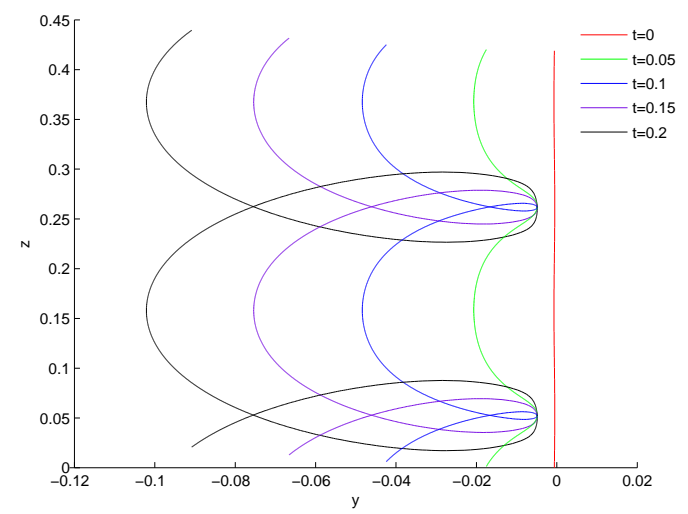

(d)

Figure 9: Non-Linear evolution of a perturbed rectilinear dislocation when $\sigma_{13}=20 \pi$. (a) 3 -D plot for the evolution; (b) projection onto the $x-y$ plane (c) projection onto the $x-z$ plane; (d) projection onto the $y$ - $z$ plane.

stress. To be precise, for a plane with normal $\boldsymbol{\beta}$ and a dislocation with unit Burgers vector $\hat{\mathbf{b}}$, the resolved shear stress, also known as the Schmid stress, is given by

$$
\tau=\left(\boldsymbol{\sigma}_{\mathrm{ext}} \cdot \hat{\mathbf{b}}\right) \cdot \boldsymbol{\beta},
$$

where $\boldsymbol{\sigma}_{\text {ext }}$ is the applied stress. The slip planes for the expanding blue curves are given by maximising $\tau$ with respect to $\beta$ subject to $\boldsymbol{\beta} \cdot \mathbf{b}=0$ and $|\boldsymbol{\beta}|=1$. For the parameters used in the simulations presented this gives $\boldsymbol{\beta}=(0.9950,0,-0.0995)^{T}$, which is in agreement with Fig. 9. Other simulations (not shown) confirm this result.

One way to understand this evolution is to imagine the initial rectilinear mixed dislocation to be divided into a sequence of edge and screw components. The screw components then cross slip and bow out, while the edge components rotate to accommodate this.

We would expect the vertical separation between the family of parallel slip planes in Figure 10 to be the wavelength of the instability. The normal separation between these planes is 


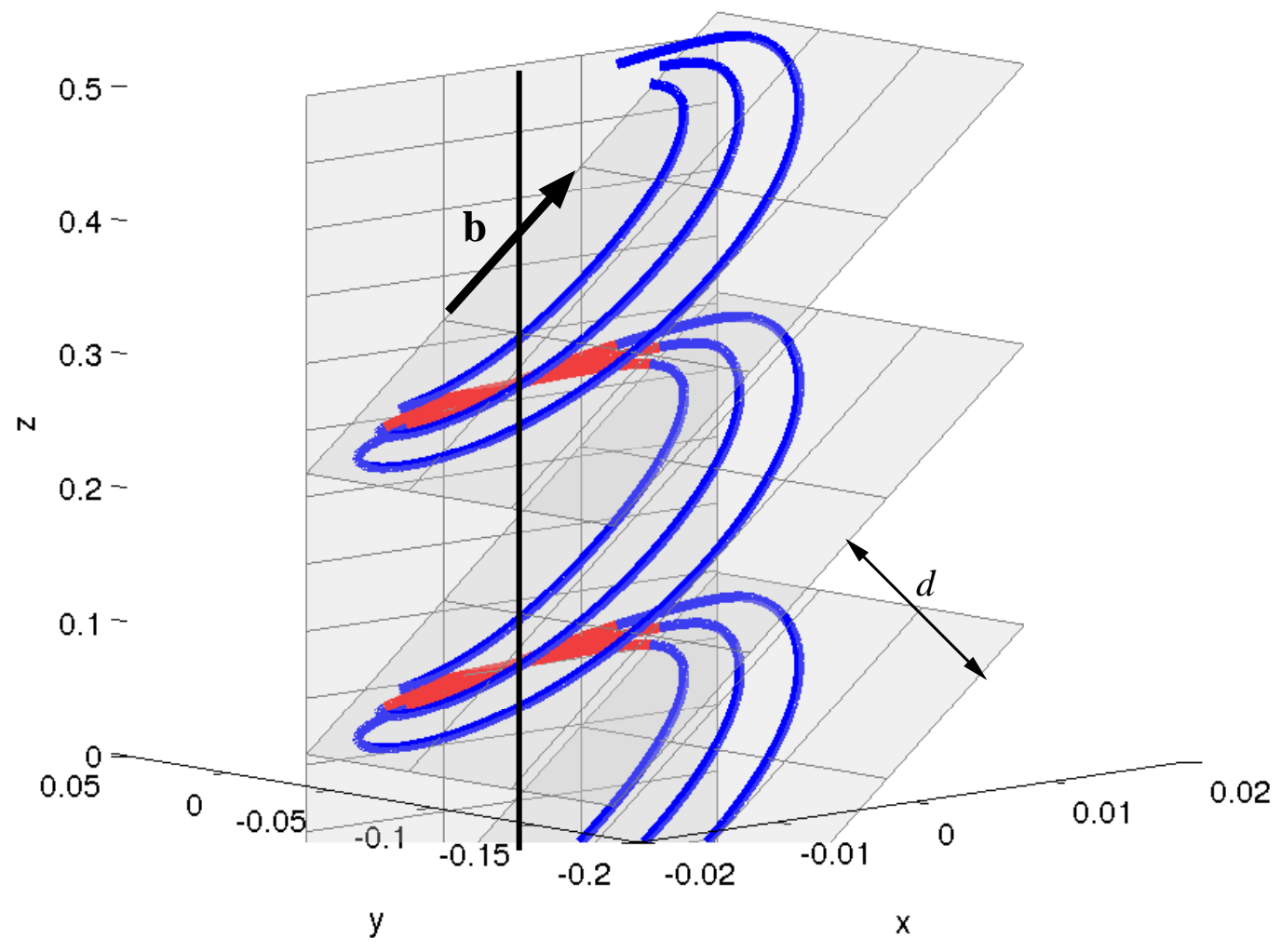

Figure 10: Schematic diagram of the non-linear evolution. The black vertical line shows the initial position of the dislocation. The normal separation between the slip planes on which the blue dislocation segments expand is $d$.

then $\hat{b}_{1}$ times this. Redimensionalising, this gives a normal separation between slip planes of

$$
d=\frac{2 \pi b_{1} L}{k b}=\frac{\epsilon \mu(1+\nu)(1-\phi) b_{1}}{2(1-\nu) \sigma_{13} \hat{k}} ;
$$

For general $\sigma_{23}$ we find $\hat{k}$ and $b_{1}$ by maximising (28). The most unstable mode occurs when $\sigma_{23}=0$ giving $\hat{k} \approx 0.54$ and $b_{1} \approx 0.98 \epsilon b$.

The most difficult parameter to estimate here is $\epsilon$, which in principle can be determined from atomistic simulations. If we imagine that a dislocation line slightly tilted away from the Burgers vector remains mainly screw and accounts for the deviation by making kinks with atomic height $h$, then the tilt angle $\alpha$ is related to the average separation between kinks $D$ through $\tan \alpha=h / D$. Thus dislocations can be considered as a combination of screw segments and kinks (and thus able to cross slip) as long as $\alpha<\tan ^{-1}(h / D)$ [12]. Since $\hat{b}_{1}=\sin \alpha \approx \tan \alpha$ for small $\alpha$, this gives the estimate $\epsilon \approx h / D$. In [12] the typical with of a kink is given as $5 h$, giving $\epsilon \approx 0.2$. In [20] the width of a kink is given as $22 h$, giving $\epsilon \approx 0.045$. In [30] the width of a kink is found to be about 20 Burgers vectors, corresponding to $21.2 h$, giving $\epsilon \approx 0.047$. 
Using the representative values

$$
\mu=20 \mathrm{GPa}, \quad \sigma_{13}=1 \mathrm{MPa}, \quad \nu=0.3, \quad \epsilon=0.045, \quad b=2.48 \AA,
$$

gives a spacing of $104 \AA$. Using instead $\epsilon=0.2$ would give a spacing of $2068 \AA$. We recall that the experiments of Brown [7] suggest a spacing of $200-800 \AA$.

Finally we speculate on the possible effect of lattice friction or Peierls stress, which is not included in the law of motion (6). We would expect the inclusion of a Peierls stress to increase the threshold stress for the instability to be activated. We would also expect the range of values of applied stress for which the nonlinear evolution arrests at a new equilibrium as in Fig 7 to be increased. However, we would not expect it to have an effect on the maximally unstable wavelength, and therefore we would expect the spacing between active slip planes to remain unaltered.

A related phenomenon is that in practice cross-slip is not automatic once a segment becomes of screw orientation, as we have presumed. A dislocation core is spread out over a range of 5-10 Burger's vectors, and may be localized in partial dislocations, such as the 1/2(112) Shockley partials in FCC. In order for the screw core to cross-slip, the dislocation core must be constricted (at least according the Friedel-Escaig model) and then re-assembled on any conjugate slip plane for which a reasonable Schmidt stress exists. The activation energy required to do this is likely to further increase the threshold stress for our instability. On the other hand, we note that Aluminium has a high stacking fault energy and therefore is not predisposed to forming partial dislocations easily.

\subsection{A microscopic analog of non-Schmid behavior and non-associated flow}

The generalised helical instability elucidated for the close to screw dislocation (Secs. 3.2 and 3.3) indicates one possible mechanism for an analog of non-Schmid type behavior at the microscopic level. This is a useful observation as, on occasion, yielding and flow characteristics at the single dislocation level survive averaging to macroscopic scales as shown in Bassani and Racherla [6]. The 'yield criterion' for the instability-equation (29)-contains information on the elastic modulus and deviation from pure screw orientation of the dislocation that is simply not contained in the resolved shear stress expression for slip on the cross-slip plane. Therefore, qualitatively, this must mean that the causes for the initiation and continued progress of the instability are different. A test for non-Schmid yielding on a given slip system, characterized by a slip direction $\hat{\mathbf{b}}$ and slip plane normal $\boldsymbol{\beta}$, is to compare the direction of the normal to the yield function for that slip system to that of the corresponding plastic strain rate tensor $(\hat{\mathbf{b}} \otimes \boldsymbol{\beta})_{\text {sym }}$ and check for differences; for yielding following Schmid's law, the stress-state dependence of the scalar yield function would necessarily have to be only through the resolved shear stress $\boldsymbol{\tau}=(\boldsymbol{\sigma} \cdot \hat{\mathbf{b}}) \cdot \boldsymbol{\beta}$, and consequently the two directions in question have to be the same. 
In the present context, as mentioned in Sec. 5.1, we obtain $\boldsymbol{\beta}$ from

$$
\max _{\boldsymbol{\beta},|\boldsymbol{\beta}|=1}(\boldsymbol{\sigma} \cdot \hat{\mathbf{b}}) \cdot \boldsymbol{\beta} \quad \text { subject to } \boldsymbol{\beta} \cdot \hat{\mathbf{b}}=0 .
$$

For $\hat{\mathbf{b}}$ parallel to $\mathbf{b}$ with only non-zero components $b_{1}, b_{3}$ and the only non-zero components of stress being $\sigma_{13}, \sigma_{23}$, it can be shown that $\boldsymbol{\beta}$ is parallel to the direction $\left(1-s^{2}, p\left(1+s^{2}\right),-s\left(1-s^{2}\right)\right)$ where $p=\sigma_{23} / \sigma_{13}$ and $s=b_{1} / b_{3}$.

The instability criterion (29) suggests two possible types of stress dependence for microscopic yielding:

$$
f_{1}(\boldsymbol{\sigma})=K \sigma_{13}^{2}-\sigma_{23}^{2}, \quad f_{2}(\boldsymbol{\sigma})=\frac{K \sigma_{13}^{2}}{\sigma_{23}^{2}} .
$$

It can be checked that the tensors $\partial f_{1} / \partial \boldsymbol{\sigma}$ and $\partial f_{2} / \partial \boldsymbol{\sigma}$ are not parallel to $(\hat{\mathbf{b}} \otimes \boldsymbol{\beta})_{\text {sym }}$, and therefore we have a microscopic analog of non-Schmid yielding and non-associated flow.

In closing we make two further points. First, with respect to the experimental observations of Cahn [10] (1951), discussed further by Mott (1951) [25], related to bending of the slip traces, it is quite conceivable that when the loops on the cross-slip plane have expanded to a significant extent they would have produced extended close-to-screw segments and if the loading orientation was such that the original plane from which cross-slip first ensued had some resolved shear stress on it, these screw segments could double cross-slip back onto the original plane producing a Frank-Read source mechanism.

Second, in the formulation of mesoscale plasticity models it is currently fashionable to introduce scalar dislocation densities with associated pde for their evolution. We believe that much like vortices in fluid dynamics and superconductivity, and as evidenced by the example considered in this paper, a great deal of fundamental physics of dislocation motion is encoded in the conservation statement of topological charge carried by a line density, a statement which is quite different in content and implications than those that arise for scalar densities.

Acknowledgements This work was initiated while AA visited the Oxford Centre for Industrial and Applied Mathematics supported by EPSRC grant EP/G015864/1. YZ is supported by EPSRC grant EP/D048400/1. We would like to thank the (anonymous) referees for many helpful comments and suggestions.

\section{References}

[1] A. Acharya. New inroads in an old subject: Plasticity, from around the atomic to the macroscopic scale. J. Mech. Phys. Sol., 58:766-778, 2010.

[2] R. J. Amodeo and N. M. Ghoniem. Dislocation dynamics. i. a proposed methodology for deformation micromechanics. Phys. Rev. B, 41(10):6958-6967, 1990. 
[3] R. Asaro. Micromechanics of crystals and polycrystals. Adv. Appl. Mech., 23:1-115, 1983.

[4] R. J. Asaro and J. R. Rice. Strain localization in ductile single crystals. J. Mech. Phys. Solids, 25:309-338, 1977.

[5] J. Bassani. Plastic flow of crystals. Adv. Appl. Mech., 30:191-258, 1993.

[6] J. Bassani and V. Racherla. From non-planar dislocation cores to non-associated plasticity and strain bursts. Progress in Materials Science, 56(6):852-863, 2011.

[7] A. F. Brown. Fine structure of slip-zones. Nature, 163:961-962, 1949.

[8] J. M. Burgers. Some considerations of the field of stress connected with dislocations in a regular crystal lattice. Part I. Proc. Kon. Nederl. Akad. Wetensch., 42:293-325, 1939.

[9] J. M. Burgers. Some considerations of the field of stress connected with dislocations in a regular crystal lattice. Part II. Proc. Kon. Nederl. Akad. Wetensch., 42:378-399, 1939.

[10] R. W. Cahn. Slip and polygonization in aluminium. J. Inst. Met., 79:129-158, 1951.

[11] W. Cai, A. Arsenlis, C. Weinberger, and V. Bulatov. A non-singular continuum theory of dislocations. J. Mech. Phys. Solids, 54(3):561-587, 2006.

[12] W. Cai and V. V. Bulatov. Mobility laws in dislocation dynamics simulations. Mat. Sci. Eng. A, 387389:277281, 2004.

[13] S. J. Chapman. A mean-field model of superconducting vortices in three dimensions. SIAM J. Appl. Math, 55:1259-1274, 1995.

[14] S. J. Chapman. A hierarchy of models for type-II superconductors. SIAM Review, 42(4):555-598, 2000.

[15] S. J. Chapman and G. Richardson. Motion of vortices in type-II superconductors. SIAM J. Appl. Math., 55(5):1275-1296, 1995.

[16] S. Gavazza and D. Barnett. The self-force on a planar dislocation loop in an anisotropic linear-elastic medium. Journal of the Mechanics and Physics of Solids, 24(4):171 - 185, 1976.

[17] R. D. Heidenreich and W. Shockley. Electron microscope and electron-diffraction study of slip in metal crystals. J. Appl. Phys., 18:1029-1031, 1947.

[18] J. P. Hirth, H. M. Zbib, and J. Lothe. Forces on high velocity dislocations. Modelling Simul. Mater. Sci. Eng., 6:165169, 1998).

[19] D. Hull and D. J. Bacon. Introduction to Dislocations. Elsevier, fifth edition, 2011.

[20] J.Hirth and J.Lothe. Theory of Dislocations. Wiley, New York, 2nd edition, 1982.

[21] G. Jiang and D. Peng. Weighed ENO schemes for Hamilton-Jacobi equations. SIAM J. Sci. Comp., 21(6):2126-2143, 2000. 
[22] E. Kröner. Kontinnuumstheorie der bersetzungen und eigenspannungen. Springer, Berlin, 1958.

[23] L. P. Kubin, G. Canova, M. Condat, B. Devincre, V. Pontikis, and Y. Brechet. Dislocation microstructures and plastic flow: a 3d simulation. Solid State Phenomena, 23-24:455-472, 1992.

[24] J. Marian, W. Cai, and V. V. Bulatov. Dynamic transitions from smoo= th to rough to twinning in dislocation motion. Nature Materials, 3(158), 2004.

[25] N. F. Mott. The mechanical properties of metals. Proc. Phys. Soc. B, 64:729-742, 1951.

[26] M. Ortiz and E. A. Repetto. Nonconvex energy minimization and dislocation structures in ductile single crystals. J. Mech. Phys. Solids, 47(2):397-462, 1999.

[27] M. Peach and J. S. Koehler. The forces excerted on dislocations and the stress fields produced by them. Phys. Rev., 80:436-439, 1950.

[28] J. R. Rice. The localization of plastic deformation. In W. T. Koiter, editor, Theoretical and Applied Mechanics (Proceedings of the 14th International Congress on Theoretical and Applied Mechanics, Delft, 1976), volume 1, pages 207-220. North-Holland Publishing Co., 1976.

[29] G. Richardson. Instability of a superconducting line vortex. Physica D, 110:139-153, 1997.

[30] L. Ventelon, F. Willaime, and P. Leyronnas. Atomistic simulation of single kinks of screw dislocations in $\alpha$-fe. J. Nucl. Mat., 386388:2629, 2009.

[31] Y. Xiang, L.-T. Cheng, D. J. Srolovitz, and W. E. A level set method for dislocation dynamics. Acta Materialia, 51(18):5499-5518, 2003.

[32] D. G. Zhao, H. Q. Wang, and Y. Xiang. Asymptotic behaviors of the stress fields in the vicinity of dislocations and dislocation segments. Philosophical Magazine, 92(18):2351$2374,2012$.

\section{Appendix}

\subsection{Local expansion of $\sigma$}

Using the fact that

$$
\frac{1}{Z^{3}} \sim \frac{1}{\varepsilon^{3}} \frac{1}{\left(R^{2}+l^{2}\right)^{3 / 2}}+\frac{1}{\varepsilon^{2}} \frac{3 \kappa R \cos \theta l^{2}}{2\left(R^{2}+l^{2}\right)^{5 / 2}}+\mathcal{O}\left(\frac{1}{\varepsilon}\right)
$$

the expansion for $\boldsymbol{\sigma}^{1}$ can be derived in terms of local coordinates. Instead of giving the cumbersome details of all the calculations, we only list the possible singular terms. From (12), we can see that the projection from dq to $\mathbf{m}$ is $\mathcal{O}\left(\varepsilon^{3}\right)$, which makes all the terms arising 
from dyadic products with $\mathbf{m}$ in the expression for $\boldsymbol{\sigma}^{1}$ regular. Therefore, only the remaining six entries for the second order tensor in the local coordinate system need to be considered, and these are:

$$
\begin{aligned}
\sigma_{n n} & \sim-\int_{-S / \varepsilon}^{S / \varepsilon} \frac{\hat{b}_{m} \kappa l^{2}}{\left(R^{2}+l^{2}\right)^{3 / 2}} \mathrm{~d} l+\mathcal{O}(1)=2 \hat{b}_{m} \kappa \log (R \varepsilon)+\mathcal{O}(1) \\
\sigma_{n l} & \sim-\frac{1}{\varepsilon} \int_{-S / \varepsilon}^{S / \varepsilon} \frac{\hat{b}_{l} R \sin \theta}{\left(R^{2}+l^{2}\right)^{3 / 2}} \mathrm{~d} l+\mathcal{O}(1)=-\frac{2}{R \varepsilon} \hat{b}_{l} \sin \theta+\mathcal{O}(1) \\
\sigma_{m n} & \sim \int_{-S / \varepsilon}^{S / \varepsilon} \frac{\kappa\left(\hat{b}_{l} R \cos \theta+\hat{b}_{n} l\right)}{\left(R^{2}+l^{2}\right)^{3 / 2}} l \mathrm{~d} l+\mathcal{O}(1)=-2 \hat{b}_{n} \kappa \log (R \varepsilon)+\mathcal{O}(1) \\
\sigma_{m l} & \sim \frac{1}{\varepsilon} \int_{-S / \varepsilon}^{S / \varepsilon} \frac{\hat{b}_{l} R \cos \theta+\hat{b}_{n} l}{\left(R^{2}+l^{2}\right)^{3 / 2}} \mathrm{~d} l-\frac{1}{2} \int_{-S / \varepsilon}^{S / \varepsilon} \frac{\hat{b}_{l} \kappa l^{2}}{\left(R^{2}+l^{2}\right)^{3 / 2}} \mathrm{~d} l+\mathcal{O}(1) \\
& =\frac{2}{R \varepsilon} \hat{b}_{l} \cos \theta+\hat{b}_{l} \kappa \log (R \varepsilon)+\mathcal{O}(1), \\
\sigma_{l n} & \sim \int_{-S / \varepsilon}^{S / \varepsilon} \frac{\hat{b}_{n} R \sin \theta \kappa}{\left(R^{2}+l^{2}\right)^{3 / 2}} l \mathrm{~d} l+\mathcal{O}(1)=\mathcal{O}(1), \\
\sigma_{l l} & \sim \frac{1}{\varepsilon} \int_{-S / \varepsilon}^{S / \varepsilon} \frac{\hat{b}_{n} R \sin \theta-\hat{b}_{m} R \cos \theta}{\left(R^{2}+l^{2}\right)^{3 / 2}} \mathrm{~d} l+\frac{1}{2} \int_{-S / \varepsilon}^{S / \varepsilon} \frac{\hat{b}_{m} \kappa l^{2}}{\left(R^{2}+l^{2}\right)^{3 / 2}} \mathrm{~d} l+\mathcal{O}(1) \\
& \sim \frac{2}{R \varepsilon}\left(\hat{b}_{n} \sin \theta-\hat{b}_{m} \cos \theta\right)-\hat{b}_{m} \kappa \log (R \varepsilon)+\mathcal{O}(1) .
\end{aligned}
$$

Thus the expansion $\boldsymbol{\sigma}^{\mathbf{1}}$ in the local basis $(\mathbf{n}, \mathbf{m}, \mathbf{l})$ is

$$
\boldsymbol{\sigma}^{1} \sim \frac{2}{\varepsilon R}\left(\begin{array}{ccc}
0 & 0 & -\hat{b}_{l} \sin \theta \\
0 & 0 & \hat{b}_{l} \cos \theta \\
0 & 0 & \hat{b}_{n} \sin \theta-\hat{b}_{m} \cos \theta
\end{array}\right)+\log (R \varepsilon) \kappa\left(\begin{array}{ccc}
2 \hat{b}_{m} & 0 & 0 \\
-2 \hat{b}_{n} & 0 & \hat{b}_{l} \\
0 & 0 & -\hat{b}_{m}
\end{array}\right)+\cdots
$$

as $\varepsilon \rightarrow 0$.

Let us now consider $\boldsymbol{\sigma}^{2}$. By using the fact that

$$
\frac{\partial^{2} Z}{\partial q_{i} \partial q_{j}}=\delta_{i j} \frac{1}{Z}-\frac{z_{i} z_{j}}{Z^{3}}
$$

where $z_{i}$ is the $i$ th entry of $\mathbf{z}$ and $\delta_{i j}$ is the Kronecker delta, we can rewrite $\boldsymbol{\sigma}^{2}$ as

$$
\begin{aligned}
(1-\nu) \boldsymbol{\sigma}^{2}= & \int_{-S / \varepsilon}^{S / \varepsilon}(\mathrm{d} \mathbf{q} \cdot(\hat{\mathbf{b}} \wedge \nabla))\left((\nabla \otimes \nabla) Z-\frac{2}{Z} \mathbf{I}\right) \\
& =-\int_{-S / \varepsilon}^{S / \varepsilon} \mathrm{d} \mathbf{q} \cdot\left(\hat{\mathbf{b}} \wedge \nabla \frac{1}{Z}\right) \mathbf{I}-\int_{-S / \varepsilon}^{S / \varepsilon}(\mathrm{d} \mathbf{q} \cdot(\hat{\mathbf{b}} \wedge \nabla))\left(\frac{\mathbf{z} \otimes \mathbf{z}}{Z^{3}}\right) .
\end{aligned}
$$


From the first term of (38), we obtain

$$
\begin{aligned}
\int_{-S / \varepsilon}^{S / \varepsilon} \frac{\mathrm{d} \mathbf{q}}{Z^{3}} & \cdot(\hat{\mathbf{b}} \wedge \mathbf{z}) \mathbf{I} \\
& \sim-\frac{1}{\varepsilon} \int_{-S / \varepsilon}^{S / \varepsilon} \frac{\hat{b}_{n} R \sin \theta-\hat{b}_{m} R \cos \theta}{\left(R^{2}+l^{2}\right)^{3 / 2}} \mathrm{~d} l \mathbf{I}+\frac{1}{2} \int_{-S / \varepsilon}^{S / \varepsilon} \frac{\hat{b}_{m} \kappa l^{2}}{\left(R^{2}+l^{2}\right)^{3 / 2}} \mathrm{~d} l \mathbf{I}+\mathcal{O}(1) \\
& =-\frac{2}{R \varepsilon}\left(\hat{b}_{n} \sin \theta-\hat{b}_{m} \cos \theta\right) \mathbf{I}-\hat{b}_{m} \kappa \log (R \varepsilon) \mathbf{I}+\mathcal{O}(1)
\end{aligned}
$$

The second term of (38) can rewritten

$$
\begin{aligned}
-\int_{-S / \varepsilon}^{S / \varepsilon} & (\mathrm{d} \mathbf{q} \cdot(\hat{\mathbf{b}} \wedge \nabla))\left(\frac{1}{Z^{3}}(\mathbf{z} \otimes \mathbf{z})\right) \\
& =-\int_{-S / \varepsilon}^{S / \varepsilon}(\mathrm{d} \mathbf{q} \cdot(\hat{\mathbf{b}} \wedge \nabla)) \frac{\mathbf{z} \otimes \mathbf{z}}{Z^{3}}-\int_{-S / \varepsilon}^{S / \varepsilon}\left(\frac{\mathrm{d} \mathbf{q}}{Z^{3}} \cdot(\hat{\mathbf{b}} \wedge \nabla)\right)(\mathbf{z} \otimes \mathbf{z}) \\
& =\int_{-S / \varepsilon}^{S / \varepsilon} \frac{3 \mathrm{~d} \mathbf{q}}{Z^{5}} \cdot(\hat{\mathbf{b}} \wedge \mathbf{z})(\mathbf{z} \otimes \mathbf{z})-\int_{-S / \varepsilon}^{S / \varepsilon}\left(\frac{\mathrm{d} \mathbf{q}}{Z^{3}} \cdot(\hat{\mathbf{b}} \wedge \nabla)\right)(\mathbf{z} \otimes \mathbf{z}) \\
& =\boldsymbol{\sigma}^{21}+\boldsymbol{\sigma}^{22}
\end{aligned}
$$

say. From the expansion

$$
\frac{\mathrm{d} \mathbf{q}}{Z^{5}} \cdot(\hat{\mathbf{b}} \wedge \mathbf{z}) \sim-\frac{1}{\varepsilon^{3}} \frac{\left(\hat{b}_{n} R \sin \theta-\hat{b}_{m} \cos \theta\right) \mathrm{d} l}{\left(R^{2}+l^{2}\right)^{5 / 2}}+\frac{1}{\varepsilon^{2}} \frac{\hat{b}_{m} \kappa l^{2} \mathrm{~d} l}{\left(R^{2}+l^{2}\right)^{5 / 2}}+\mathcal{O}\left(\frac{1}{\varepsilon}\right),
$$


$\boldsymbol{\sigma}^{21}$ can be calculated term by term in a similar way to $\boldsymbol{\sigma}^{1}$, giving

$$
\begin{aligned}
\sigma_{n n} & \sim-\frac{3}{\varepsilon} \int_{-S / \varepsilon}^{S / \varepsilon} \frac{\left(\hat{b}_{n} \sin \theta-\hat{b}_{m} \cos \theta\right) R^{3} \cos ^{2} \theta}{\left(R^{2}+l^{2}\right)^{5 / 2}} \mathrm{~d} l+\mathcal{O}(1) \\
& =-\frac{4 \cos ^{2} \theta\left(\hat{b}_{n} \sin \theta-\hat{b}_{m} \cos \theta\right)}{R \varepsilon}+\mathcal{O}(1), \\
\sigma_{m n}=\sigma_{n m} & \sim-\frac{3}{\varepsilon} \int_{-S / \varepsilon}^{S / \varepsilon} \frac{\left(\hat{b}_{n} \sin \theta-\hat{b}_{m} \cos \theta\right) R^{3} \cos \theta \sin \theta}{\left(R^{2}+l^{2}\right)^{5 / 2}} \mathrm{~d} l+\mathcal{O}(1) \\
& =-\frac{4 \cos \theta \sin \theta\left(\hat{b}_{n} \sin \theta-\hat{b}_{m} \cos \theta\right)}{R \varepsilon}+\mathcal{O}(1), \\
\sigma_{n l}=\sigma_{l n} & \sim 3 \int_{-S / \varepsilon}^{S / \varepsilon} \frac{\hat{b}_{l} \kappa R^{2} \cos \theta \sin \theta}{\left(R^{2}+l^{2}\right)^{5 / 2}} l^{2} \mathrm{~d} l+\mathcal{O}(1)=\mathcal{O}(1), \\
\sigma_{m m} & \sim-\frac{3}{\varepsilon} \int_{-S / \varepsilon}^{S / \varepsilon} \frac{\left(\hat{b}_{n} \sin \theta-\hat{b}_{m} \cos \theta\right) R^{3} \sin ^{2} \theta}{\left(R^{2}+l^{2}\right)^{5 / 2}} \mathrm{~d} l+\mathcal{O}(1) \\
& =-\frac{4 \sin ^{2} \theta\left(\hat{b}_{n} \sin \theta-\hat{b}_{m} \cos \theta\right)}{R \varepsilon}+\mathcal{O}(1), \\
\sigma_{m l}=\sigma_{l m} & \sim 3 \int_{-S / \varepsilon}^{S / \varepsilon} \frac{\hat{b}_{l} \kappa R^{2} \sin { }^{2} \theta}{\left(R^{2}+l^{2}\right)^{5 / 2}} l^{2} \mathrm{~d} l+\mathcal{O}(1)=\mathcal{O}(1), \\
\sigma_{l l} & \sim-\frac{3}{\varepsilon} \int_{-S / \varepsilon}^{S / \varepsilon} \frac{\left(\hat{b}_{n} \sin \theta-\hat{b}_{m} \cos \theta\right) R l^{2}}{\left(R^{2}+l^{2}\right)^{5 / 2}} \mathrm{~d} l+\frac{3}{2} \int_{-S / \varepsilon}^{S / \varepsilon} \frac{\hat{b}_{m} \kappa l^{4}}{\left(R^{2}+l^{2}\right)^{5 / 2}} \mathrm{~d} l+\mathcal{O}(1) \\
& =-\frac{2\left(\hat{b}_{n} \sin \theta-\hat{b}_{m} \cos \theta\right)}{R \varepsilon}-3 \hat{b}_{m} \kappa \log (R \varepsilon)+\mathcal{O}(1) .
\end{aligned}
$$

Thus

$$
\begin{array}{r}
\boldsymbol{\sigma}^{21} \sim-\frac{4}{R \varepsilon}\left(\hat{b}_{n} \sin \theta-\hat{b}_{m} \cos \theta\right)\left(\begin{array}{ccc}
\cos ^{2} \theta & \cos \theta \sin \theta & 0 \\
\cos \theta \sin \theta & \sin ^{2} \theta & 0 \\
0 & 0 & 1 / 2
\end{array}\right) \\
+\kappa \log (R \varepsilon)\left(\begin{array}{ccc}
0 & 0 & 0 \\
0 & 0 & 0 \\
0 & 0 & -3 \hat{b}_{m}
\end{array}\right)+\mathcal{O}(1) .
\end{array}
$$

For $\boldsymbol{\sigma}^{\mathbf{2 2}}$ we first note that if the unit vector in the $x_{i}$ direction is denoted by $\mathbf{e}_{i}$, then we have

$$
\begin{aligned}
{[\mathrm{d} \mathbf{q} \cdot(\hat{\mathbf{b}} \wedge} & \left.\left.\nabla^{\prime}(\mathbf{z} \otimes \mathbf{z})\right)\right]_{i, j}=2\left(\left(\mathbf{z} \cdot \mathbf{e}_{i}\right)\left(\hat{\mathbf{b}} \wedge \mathbf{e}_{j}\right) \cdot \mathrm{d} \mathbf{q}\right)^{S} \\
& =2\left(\left(\mathbf{z} \cdot \mathbf{e}_{i}\right)\left(\hat{b}_{n} \mathbf{n}+b_{m} \mathbf{m}+\hat{b}_{l} \mathbf{l}\right) \wedge\left(n_{j} \mathbf{n}+m_{j} \mathbf{m}+t_{j} \mathbf{l}\right) \cdot\left(\varepsilon \mathbf{l}+\varepsilon^{2} \mathbf{n} \kappa l\right) \mathrm{d} l\right)^{S} \\
& \left.=2 \mathrm{~d} l\left(z_{i}\left(\varepsilon\left(\hat{b}_{n} m_{j}-b_{m} n_{j}\right)+\varepsilon^{2} \kappa l\left(b_{m} t_{j}-\hat{b}_{l} m_{j}\right)\right)\right)\right)^{S} \\
& =2\left(\varepsilon \hat{b}_{n}-\varepsilon^{2} \hat{b}_{l} \kappa l\right) \mathrm{d} l\left(z_{i} m_{j}\right)^{S}-2 \varepsilon b_{m} \mathrm{~d} l\left(z_{i} n_{j}\right)^{S}+2 \varepsilon^{2} b_{m} \kappa l \mathrm{~d} l\left(z_{i} t_{j}\right)^{S},
\end{aligned}
$$


where ${ }^{S}$ denotes the symmetric part and $n_{j}, m_{j}, t_{j}$ and $z_{j}$ are the $j$-th entries of the corresponding vectors. Therefore the integrand of $\boldsymbol{\sigma}^{\mathbf{2 2}}$ can be written as

$$
\begin{aligned}
-\mathrm{d} \mathbf{q} \cdot \frac{1}{Z^{3}}(\hat{\mathbf{b}} \wedge & \nabla(\mathbf{z} \otimes \mathbf{z}))=\frac{\mathrm{d} l}{Z^{3}}\left(-\varepsilon \hat{b}_{n}+\varepsilon^{2} \hat{b}_{l} \kappa l\right)((\mathbf{z} \otimes \mathbf{m})+(\mathbf{m} \otimes \mathbf{z})) \\
+ & \frac{\varepsilon \hat{b}_{m} \mathrm{~d} l}{Z^{3}}((\mathbf{z} \otimes \mathbf{n})+(\mathbf{n} \otimes \mathbf{z}))-\frac{\varepsilon^{2} \hat{b}_{m} \kappa l}{Z^{3}}((\mathbf{z} \otimes \mathbf{l})+(\mathbf{l} \otimes \mathbf{z})) .
\end{aligned}
$$

With the expansion

$$
-\frac{1}{Z^{3}}\left(\varepsilon \hat{b}_{n}-\varepsilon^{2} \hat{b}_{l} \kappa l\right) \sim-\frac{1}{\varepsilon^{2}} \frac{\hat{b}_{n}}{\left(R^{2}+l^{2}\right)^{3 / 2}}-\frac{1}{\varepsilon} \frac{3 \hat{b}_{n} \kappa R \cos \theta l^{2}}{2\left(R^{2}+l^{2}\right)^{5 / 2}}+\frac{1}{\varepsilon} \frac{\hat{b}_{l} \kappa l}{\left(R^{2}+l^{2}\right)^{3 / 2}}+\cdots,
$$

the singular terms in $\boldsymbol{\sigma}^{\mathbf{2 2}}$ can be evaluated as the sum of a stress tensor and its transpose. This stress tensor has entries

$$
\begin{aligned}
\sigma_{n m} & \sim \frac{1}{\varepsilon} \int_{-S / \varepsilon}^{S / \varepsilon} \frac{\hat{b}_{n} R \cos \theta}{\left(R^{2}+l^{2}\right)^{3 / 2}} \mathrm{~d} l-\frac{1}{2} \int_{-S / \varepsilon}^{S / \varepsilon} \frac{\hat{b}_{n} \kappa l^{2}}{\left(R^{2}+l^{2}\right)^{3 / 2}} \mathrm{~d} l+\frac{3}{2} \int_{-S / \varepsilon}^{S / \varepsilon} \frac{\hat{b}_{n} \kappa R^{2} \cos ^{2} \theta}{\left(R^{2}+l^{2}\right)^{5 / 2}} l^{2} \mathrm{~d} l+o(1) \\
& =\frac{2}{R \varepsilon} \hat{b}_{n} \cos \theta+\hat{b}_{n} \kappa \log (R \varepsilon)+\mathcal{O}(1) \\
\sigma_{m m} & \sim \frac{1}{\varepsilon} \int_{-S / \varepsilon}^{S / \varepsilon} \frac{\hat{b}_{n} R \sin \theta}{\left(R^{2}+l^{2}\right)^{3 / 2}} \mathrm{~d} l+\frac{3}{2} \int_{-S / \varepsilon}^{S / \varepsilon} \frac{\hat{b}_{n} \kappa R^{2} \cos \theta \sin \theta}{\left(R^{2}+l^{2}\right)^{5 / 2}} l^{2} \mathrm{~d} l+\mathcal{O}(1) \\
& =\frac{2}{R \varepsilon} \hat{b}_{n} \sin \theta+\mathcal{O}(1) \\
\sigma_{l m} & \sim \int_{-S / \varepsilon}^{S / \varepsilon} \frac{\hat{b}_{l} \kappa l^{2}}{\left(R^{2}+l^{2}\right)^{3 / 2}} \mathrm{~d} l+\mathcal{O}(1)=-2 \hat{b}_{l} \kappa \log (R \varepsilon)+\mathcal{O}(1) \\
\sigma_{n n} & \sim-\frac{1}{\varepsilon} \int_{-S / \varepsilon}^{S / \varepsilon} \frac{\hat{b}_{m} R \cos \theta}{\left(R^{2}+l^{2}\right)^{3 / 2}} \mathrm{~d} l+\frac{1}{2} \int_{-S / \varepsilon}^{S / \varepsilon} \frac{\hat{b}_{m} \kappa l^{2}}{\left(R^{2}+l^{2}\right)^{3 / 2}} l^{2} \mathrm{~d} l+\mathcal{O}(1) \\
& =-\frac{2}{R \varepsilon} \hat{b}_{m} \cos \theta-\hat{b}_{m} \kappa \log (R \varepsilon)+\mathcal{O}(1), \\
\sigma_{m n} & \sim-\frac{1}{\varepsilon} \int_{-S / \varepsilon}^{S / \varepsilon} \frac{\hat{b}_{m} R \sin \theta}{\left(R^{2}+l^{2}\right)^{3 / 2}} \mathrm{~d} l+\mathcal{O}(1)=-\frac{2}{R \varepsilon} \hat{b}_{m} \sin \theta+\mathcal{O}(1) \\
\sigma_{l l} & \sim \int_{-S / \varepsilon}^{S / \varepsilon} \frac{\hat{b}_{m} \kappa l^{2}}{\left(R^{2}+l^{2}\right)^{3 / 2}} \mathrm{~d} l+\mathcal{O}(1)=-2 \hat{b}_{m} \kappa \log (R \varepsilon)+\mathcal{O}(1)
\end{aligned}
$$

Thus the expression for $\boldsymbol{\sigma}^{22}$ can be obtained as

$$
\begin{array}{r}
\sigma^{22} \sim \frac{2}{R \varepsilon}\left(\begin{array}{ccc}
-2 \hat{b}_{m} \cos \theta & \hat{b}_{n} \cos \theta-\hat{b}_{m} \sin \theta & 0 \\
\hat{b}_{n} \cos \theta-\hat{b}_{m} \sin \theta & 2 \hat{b}_{n} \sin \theta & 0 \\
0 & 0 & 0
\end{array}\right) \\
+\kappa \log (R \varepsilon)\left(\begin{array}{ccc}
-2 \hat{b}_{m} & \hat{b}_{n} & 0 \\
\hat{b}_{n} & 0 & -2 \hat{b}_{l} \\
0 & -2 \hat{b}_{l} & -4 \hat{b}_{m}
\end{array}\right)+\cdots
\end{array}
$$




\subsection{Derivation of equation (25)}

For there to exist non-zero solutions of $(22)$ the determinant of the matrix must vanish, so that

$$
\left(\lambda+c_{1} k^{2}\right)^{2}\left(\lambda+c_{2} k^{2}\right)^{2}+2 a_{12} a_{21}\left(\lambda+c_{1} k^{2}\right)\left(\lambda+c_{2} k^{2}\right) k^{2}+a_{22}^{2}\left(\lambda+c_{1} k^{2}\right) k^{2}+a_{12}^{2} a_{21}^{2} k^{4}=0 .
$$

Completing the square gives

$$
\left(\left(\lambda+c_{1} k^{2}\right)\left(\lambda+c_{2} k^{2}\right)+a_{12} a_{21} k^{2}\right)^{2}+a_{22} k^{2}\left(\lambda+c_{1} k^{2}\right)^{2}=0,
$$

so that

$$
\left(\lambda+c_{1} k^{2}\right)\left(\lambda+c_{2} k^{2}\right)+a_{12} a_{21} k^{2}=\mp \mathrm{i} a_{22} k\left(\lambda+c_{1} k^{2}\right) .
$$

Now collecting powers of $\lambda$ gives

$$
\lambda^{2}+\left(\left(c_{1}+c_{2}\right) k^{2} \pm \mathrm{i} a_{22} k\right) \lambda+c_{1} c_{2} k^{4}+a_{12} a_{21} k^{2} \pm \mathrm{i} a_{22} c_{1} k^{3}=0 .
$$

The solutions for $\lambda$ are therefore

$$
\lambda=\frac{1}{2}\left(-\left(c_{1}+c_{2}\right) k^{2} \mp \mathrm{i} a_{22} k \pm \sqrt{\Delta}\right),
$$

where

$$
\Delta=\left(c_{1}-c_{2}\right)^{2} k^{4}-\left(a_{22}^{2}+4 a_{12} a_{21}\right) k^{2} \pm 2 \mathrm{i} a_{22}\left(c_{2}-c_{1}\right) k^{3} .
$$

Now observing that, for real $x$ and $y$,

$$
(x+\mathrm{i} y)^{1 / 2}=\frac{1}{\sqrt{2}}\left(\sqrt{\sqrt{x^{2}+y^{2}}+x}+\mathrm{i} \sqrt{\sqrt{x^{2}+y^{2}}-x}\right),
$$

we find that the real part of $\lambda$ is given by

$$
\begin{aligned}
\operatorname{Re}(\lambda)= & -\frac{\left(c_{1}+c_{2}\right) k^{2}}{2} \\
& \pm \frac{k}{2 \sqrt{2}}\left(\sqrt{\left(c_{1}-c_{2}\right)^{4} k^{4}+2\left(c_{1}-c_{2}\right)^{2}\left(a_{22}^{2}-4 a_{12} a_{21}\right) k^{2}+\left(a_{22}^{2}+4 a_{12} a_{21}\right)^{2}}\right. \\
& \left.\quad+\left(c_{1}-c_{2}\right)^{2} k^{2}-\left(a_{22}^{2}+4 a_{12} a_{21}\right)\right)^{1 / 2} .
\end{aligned}
$$

Substituting for $c_{1}, c_{2}, a_{12}, a_{21}$ and $a_{22}$ leads to (25). 